ARTICLE OPEN

Check for updates

\title{
Exosomal transfer of tumor-associated macrophage-derived hsa_circ_0001610 reduces radiosensitivity in endometrial
}

\section{cancer}

\author{
Xiaobin Gu $\mathbb{D}^{1 凶}{ }^{凶}$, Yonggang Shi ${ }^{1}$, Meilian Dong ${ }^{1}$, Li Jiang ${ }^{1}$, Jing Yang ${ }^{1}$ and Zheyan Liu ${ }^{1}$
}

(c) The Author(s) 2021

The occurrence of radioresistance is a clinical obstacle to endometrial cancer (EC) treatment and induces tumor relapse. In this study, we found that tumor-associated macrophages (TAMs) enriched in EC specimens were determined to present an M2-like phenotype. In vitro, the coculture of M2-polarized macrophages significantly downregulated the radiosensitivity of EC cells by releasing exosomes. Hsa_circ_0001610 was found to be abundant in exosomes derived from M2-polarized macrophages (EXOs), and hsa_circ_0001610 knockdown eliminated the reduction effect of EXOs on the radiosensitivity of EC cells. The following mechanism research revealed that hsa_circ_0001610 functioned as the competing endogenous RNA of miR-139-5p, thereby upregulating cyclin B1 expression, which is a vital pusher of radioresistance in several types of cancer by regulating the cell cycle. Hsa_circ_0001610 overexpression reduced the radiosensitivity of EC cells, which was then reversed by miR-139-5p overexpression. In vivo, the promotion effect of EXOs on xenograft tumor growth in nude mice treated with irradiation was further reinforced after hsa_circ_0001610 overexpression. In conclusion, TAM-derived exosomes transferred hsa_circ_0001610 to EC cells, and the overexpressed hsa_circ_0001610 in EC cells released cyclin B1 expression through adsorbing miR-139-5p, thereby weakening the radiosensitivity of EC cells.

Cell Death and Disease (2021)12:818; https://doi.org/10.1038/s41419-021-04087-8

\section{INTRODUCTION}

Endometrial cancer (EC) is the most frequent gynecologic malignancy, which seriously threatens the health of women worldwide [1, 2]. According to the current international guidelines, surgery combined with adjuvant therapy is the most established treatment option for patients with EC [3]. Radiotherapy, as one of the main adjuvant therapies for EC, successfully promotes the survival rate of patients with early-stage EC [4] and decreased loco-regional recurrence in patients with high-intermediate risk EC [5]. However, the occurrence of radioresistance is a clinical obstacle to EC treatment and induces tumor relapse [6]. Therefore, elucidating the mechanism of reduced radiosensitivity in EC is critical for enhancing radiotherapy potency.

Recently, the crucial role of the tumor microenvironment (TME) in tumor initiation and progression has been gradually realized. Tumor-associated macrophages (TAMs) are among the most abundant immune cells in the TME present at all stages during tumor progression. An evident TAM infiltration has been observed in human EC specimens compared with normal endometrial specimens, and the increased TAM number is positively correlated with the malignant progression of EC [7]. The research of Li et al. [8] determined that the TAMs in EC exhibited a polarized M2 phenotype. Also, Meng et al. [9] depleted TAMs in xenograft implants of melanoma cells in mice through local injection of macrophage-depleting liposomal clodronate and found that TAM depletion reinforced the antitumor effect of irradiation. These previous studies suggest that the accumulated TAMs are involved in the radioresistance of EC cells.

Increasing evidence shows that TAMs can modulate cancer progression via secreting exosomes (EXOs), a type of extracellular vesicles that mediates intercellular communication through transferring proteins, non-coding RNAs (ncRNAs), etc. from TAMs to tumor cells [10]. For instance, Feng et al. [11] reported that TAM-derived EXOs promoted intracranial aneurysm formation by delivering microRNA (miRNA)-155-5p to smooth muscle cells; Zheng et al. [12] found that TAM-derived exosomal miR-21 triggered cisplatin resistance in gastric cancer. However, whether TAM-derived EXOs could modulate the radiosensitivity of EC cells remains unclear.

Circular RNAs (circRNAs), a type of ncRNAs, have been proven to be a modulator of radiosensitivity in several cancer types, such as esophageal squamous cell carcinomas [13], nasopharyngeal carcinoma [14], and prostate cancer [15]. Utilizing RNA sequencing, $\mathrm{Xu}$ et al. [16] found that 209 circRNAs were abnormally expressed in the extracellular vesicles isolated from serum of patients with EC, suggesting that numerous circRNAs are packaged into EXOs and play a potential role in EC progression.

\footnotetext{
${ }^{1}$ Department of Radiation Oncology, The First Affiliated Hospital of Zhengzhou University, No. 1 Jianshe East Rd, Zhengzhou 450000 Henan, People's Republic of China. 冈email: fahzzugu@126.com

Edited by: Professor Stephen Tait
}

Received: 20 January 2021 Revised: 22 July 2021 Accepted: 27 July 2021

Published online: 30 August 2021 
This study determined the role of TAM-derived EXOs in the radiosensitivity of EC cells and screen out the functional exosomal circRNA, which will provide new targets for enhancing the radiosensitivity of EC cells in clinical settings.

\section{MATERIALS AND METHODS}

Tissue specimens

A total of $25 \mathrm{EC}$ specimens were obtained from patients who underwent surgical resection at The First Affiliated Hospital of Zhengzhou University. Twenty-five normal endometrial specimens obtained from patients with uterine fibroids undergoing hysterectomy were used as normal controls. No patients received preoperative chemotherapy or radiotherapy. All patients signed informed consent forms. This investigation involving human specimens was approved by the Ethics Committee of The First Affiliated Hospital of Zhengzhou University.

\section{Isolation of TAMs and tissue-resident macrophages (NTMs)}

TAMs and NTMs were obtained from human EC specimens and normal endometrial specimens, respectively, as previously described [17]. Freshly human EC specimens and normal endometrial specimens were cut into small pieces and incubated with phosphate buffer containing $30 \mu \mathrm{l}$ Liberase DL stock solution ( $28 \mathrm{U} / \mathrm{ml}$; Roche, Switzerland), $120 \mu \mathrm{l}$ Liberase TL stock solution ( $14 \mathrm{U} / \mathrm{ml}$; Roche), and $30 \mu \mathrm{l}$ DNase I (15 mg/ml; Sigma, USA) at $37^{\circ} \mathrm{C}$. Forty-five minutes later, the cell suspension was filtered using a $100 \mu \mathrm{m}$ cell strainer and centrifuged for $5 \mathrm{~min}$ at $500 \mathrm{rcf}$. The cell pellet was resuspended in $3 \mathrm{ml}$ red blood cell lysis buffer (Solarbio, China) to lyse red blood cells and centrifuged. Following this, the cell pellet was collected for qRT-PCR or resuspended in presort buffer (BD Biosciences, USA) at a density of $2 \times 10^{6}$ cells $/ \mathrm{ml}$ for flow cytometry.

\section{Generation of M2-polarized macrophages}

M2-polarized macrophages were generated from human monocytic THP-1 cells as previously described [18]. THP-1 cells (Procell Life Science \& Technology Co., Ltd., China) were incubated with phorbol 12-myristate 13acetate $(10 \mathrm{ng} / \mathrm{ml}$; MedChemExpress, USA) for $24 \mathrm{~h}$ to differentiate into M0 macrophages. Then, M2-polarized macrophages were obtained by incubation with IL-4 $(25 \mathrm{ng} / \mathrm{ml}$; Thermo Fisher, USA) and IL-13 $(25 \mathrm{ng} / \mathrm{ml}$; Thermo Fisher) for another $48 \mathrm{~h}$.

\section{Phenotypic analysis of TAMs, NTMs, and M2-polarized macrophages}

Single-cell suspensions of TNMs and TAMs were prepared as described above and stained with BUV395 anti-human-CD45, APC anti-human CD11b, FITC anti-human-CD68, or BV-480 anti-human-CD206 (all antibodies were purchased from BD Biosciences). Then, labeled cells were analyzed on a FACSCanto II Flow Cytometer (BD Biosciences), and the data were processed using FlowJo Version 10.0. For the phenotypic analysis of THP-1-derived M2-polarized macrophages, cells were stained with APC anti-human-CD11b and FITC anti-human-CD206.

In addition, immunofluorescent staining was performed on TNMs and TAMs using anti-F4/80 and anti-CD206 antibodies (all purchased from Abcam, UK).

\section{Cell culture and transfection}

Human EC cell lines Ishikawa and HEC-1B cells used in this study were purchased from Procell Life Science \& Technology Co., Ltd (China). Ishikawa cells were cultured in Dulbecco's modified Eagle's medium (DMEM) containing $10 \%$ fetal bovine serum, and HEC-1B cells were cultured in Eagle's minimum essential medium (EMEM) containing $10 \%$ fetal bovine serum in a $5 \% \mathrm{CO}_{2}$ incubator at $37^{\circ} \mathrm{C}$.

The short hairpin RNA of circ_0001610 (sh-circRNA), miR-139-5p mimic, and their negative controls (shRNA and miR-NC) were synthesized by Guangzhou Ribobio Biotechnology Co., Ltd (China). Circ_0001610 overexpression vector (circ_0001610) and its negative control (vector) were synthesized by Guangzhou Geneseed Biotechnology Co., Ltd (China). For cell transfection, Ishikawa/ HEC-1B cells were seeded in six-well plates at a density of $1 \times 10^{6}$ cells/well. When cells reached $65 \%$ confluence, shcircRNA/shRNA/circ_0001610/vector was transfected into cells using Lipofectamine $^{\text {TM }} 3000$ (Thermo Fisher, USA), and miR-139-5p mimic/ miRNC was transfected into cells using riboFECT ${ }^{\mathrm{TM}}$ CP Reagent (Guangzhou Ribobio Biotechnology Co., Ltd. China).

\section{EXOs extraction and identification}

The EXOs were isolated from the supernatant of M2-polarized macrophages cultured for $48 \mathrm{~h}$ in EXO-free culture medium. The extraction process was conducted as previously described [19]. Briefly, the supernatant was centrifuged at $2000 \times g$ for $10 \mathrm{~min}$ and $10,000 \times g$ for $1 / 2 \mathrm{~h}$ to remove dead cells and cell debris, followed by centrifugation at $110,000 \times$ g. Then, the cell pellets were resuspended in PBS and centrifuged at $110,000 \times g$ to obtain the EXOs.

The isolated EXOs were identified by transmission electron microscope (TEM) and the Western blot. For TEM, isolated EXOs were fixed with $1 \%$ glutaraldehyde, adsorbed onto a formvar/carbon-coated grid, and negatively stained with uranyl acetate solution. Then, EXOs were visualized under TEM (Hitachi, Japan).

EXO ${ }^{\text {shRNA }}$, EXO $^{\text {sh-circRNA }}$, EXO $^{\text {vector }}$, and EXO circ_0001610 $^{\text {were extracted }}$ from the culture medium of $\mathrm{M} 2$-polarized macrophages transfected with shRNA, sh-circRNA, vector, and circ_0001610 by the above method, respectively.

\section{Cell viability assay}

Ishikawa or HEC-1B cells were treated according to the corresponding protocols and then seeded in 96-well plates. Twenty-four hours later, cells were treated with $4 \mathrm{~Gy}$ irradiation at a dose of $0.5 \mathrm{~Gy} / \mathrm{min}$ (Varian2300EX, Varian, USA). Seven-two hours later, cells in each well were incubated with $10 \mu \mathrm{l}$ 3-(4,5-dimethylthiazol-2-yl)-2,5-diphenyltetrazolium bromide $(5 \mathrm{mg} /$ $\mathrm{ml}$; Beyotime Biotechnology, China) for $4 \mathrm{~h}$ and $100 \mu \mathrm{l}$ Fromazan for another $4 \mathrm{~h}$. Then, the absorbance of each well at $570 \mathrm{~nm}$ was examined using a microplate reader.

\section{Cell proliferation assay}

Cell proliferation was measured by the colony formation assay. Ishikawa or HEC-1B cells were treated according to the corresponding protocols and then seeded in six-well plates at a density of 1000 cells/well. Twenty-four hours later, cells were treated with $4 \mathrm{~Gy}$ irradiation and then cultured under a normal condition. Two weeks later, the plates were fixed with methanol and then stained with Giemsa (Beyotime Biotechnology, China). Colonies with $\geq 50$ cells were counted using an inverted microscope (Olympus Life Science, Japan).

\section{Cell apoptosis assay}

The Annexin V-FITC Apoptosis Detection Kit (Beyotime Biotechnology, China) was employed to assess cell apoptosis. Ishikawa or HEC-1B cells were treated according to the corresponding protocols and then seeded in six-well plates. Twenty-four hours later, cells were treated with 4Gy irradiation and then cultured under a normal condition for another $72 \mathrm{~h}$. Subsequently, cells were resuspended in Annexin V-FITC binding buffer at a density of $1 \times 10^{6}$ cells $/ \mathrm{ml}$ and incubated with $5 \mu \mathrm{l}$ Annexin V-FITC and $10 \mu \mathrm{l}$ propidium iodide $(\mathrm{PI})$ in the dark for $20 \mathrm{~min}$. Cell apoptosis was assessed by a FACSCanto II Flow Cytometer (BD Biosciences).

\section{Cell invasion assay}

Cell invasion was measured by the Transwell assay. Ishikawa or HEC-1B cells were treated according to the corresponding protocols, and then $2 \times$ $10^{4}$ cells were seeded in the upper chamber pre-coated with Matrigel Matrix (Corning, USA). Also, $600 \mu$ I DMEM/EMEM containing $10 \%$ FBS was added into the lower chamber. Cells that invaded the reverse side of the membrane were fixed and stained using crystal violet (Beyotime Biotechnology) after $48 \mathrm{~h}$. An inverted microscope (Olympus Life Science) was used to photograph the stained cells.

\section{Cell cycle assay}

The cell cycle was assessed using flow cytometry. Ishikawa or HEC-1B cells were treated according to the corresponding protocols and resuspended in phosphate buffer. After centrifuging, the cell pellets were fixed with ethanol overnight. On the second day, cells were stained with a staining solution containing PI (Thermo Fisher, USA) and RNAse A (Thermo Fisher) for $30 \mathrm{~min}$. Samples were analyzed by a FACSCanto II Flow Cytometer (BD Biosciences).

\section{RNA-fluorescence in situ hybridization (RNA-FISH)}

RNA-FISH was performed to examine the subcellular localization of hsa_circ_0001610 in EC cells. Ishikawa or HEC-1B cells were seeded on the coverslips. The next day, the coverslips were incubated with $4 \%$ 
paraformaldehyde and $0.5 \%$ Triton $\mathrm{X}-100$, followed by the hybridization solution containing Cy3-labeled hsa_circ_0001610 probe (produced by Guangzhou Ribo Biotechnology Co., Ltd). After counterstaining with DAPI, the coverslips were photographed using a confocal microscope.

\section{Dual-luciferase reporter assay}

The interplay between hsa_circ_0001610 and miR-139-5p was assessed by dual-luciferase reporter assay. The sequence of hsa_circ_0001610 was subcloned into the pmirGLO-basic plasmid (Promega, USA) (circ_0001610). The mutant sequence of hsa_circ_0001610 (the mutant sides were located at the potential binding sites of hsa circ 0001610 and miR-139-5p) was subcloned into the pmirGLO-basic plasmid (Mutant). Then, the well-grown HEK-293T cells were co-transfected with circ 0001610/Mutant and miR$\mathrm{NC} / \mathrm{miR}-139-5 \mathrm{p}$ mimic. Two days after transfection, the relative luciferase activities of Mutant and circ_0001610 were examined using the dualluciferase reporter assay system (Promega).

To determine the interplay between miR-139-5p and CCNB1, the CCNB1 $3^{\prime}$-untranslated region wild type (CCNB1 3'-UTR WT; containing the sequence of CCNB1 $3^{\prime}$-UTR) and CCNB1 $3^{\prime}$-UTR mutant (containing the mutant sequence of CCNB1 $3^{\prime}$-UTR and the mutant sides were located at the potential binding sites of miR-139-5p and CCNB1) were prepared. The relative luciferase activity of CCNB1 3'-UTR WT/CCNB1 $3^{\prime}$-UTR mutant in HEK-293T cells transfected with miR-NC/miR-139-5p mimic was measured as described above.

\section{Western blot}

Ishikawa, HEC-1B cells were collected, and the protein samples were extracted using RIPA lysis buffer (Shanghai Absin Biological Technology Co., Ltd, China). The expression level of cyclin B1 in protein samples was measured by Western blot as previously described [20]. To identify the isolated EXOs, protein samples were extracted from EXOs using the Exosome Protein Extraction Kit (Shanghai Hifun Biotechnology Co., Ltd. China). Then, the expression levels of CD63, CD9, and CD81 were measured by Western blot. To identify the epithelial-to-mesenchymal transition (EMT) in the tumor xenograft model mice, the protein samples were extracted from the xenograft tumor tissues, and the protein levels of zinc finger E-box binding homeobox 1 (ZEB1), N-cadherin, $\beta$-catenin, vimentin, and $\mathrm{E}$-cadherin were measured. The primary antibodies used in this study were as follows: anti-cyclin B1 antibody (1:50,000), anti-CD63 antibody (1:1000), anti-CD9 antibody (1:2000), anti-CD81 antibody (1:1000), anti-ZEB1 (1:500), $\mathrm{N}$-cadherin $(1 \mu \mathrm{g} / \mathrm{ml}), \beta$-catenin (1:5000), vimentin (1:2000), and E-cadherin $(1: 10,000)$. All antibodies were purchased from Abcam.

\section{RNA pull-down assay}

The RNA pull-down assay was employed to further confirm the binding between hsa_circ_0001610 and miR-139-5p. Guangzhou Ribobio Biotechnology Co., Ltd (China) synthesized the biotinylated miR-139-5p (BiotinmiR-139-5p) and its negative control (biotinylated control RNA, referred to as control RNA). Ishikawa and HEC-1B cells were lysed using NP40 lysis buffer (Boster Biological Technology Co., Ltd, China), followed by incubation of $20 \mathrm{nM}$ Biotin-miR-139-5p or control RNA. The biotincoupled RNA complex was pulled down by incubating with streptavidincoated magnetic beads (Thermo Fisher). The enrichment of hsa circ 0001610 in the bound fractions was examined using qRT-PCR.

\section{qRT-PCR}

Total RNA samples were isolated from TAMs, NTMs, Ishikawa cells, HEC-1B cells, and xenotransplanted tumors using Trozol (Thermo Fisher). TaqMan MicroRNA assays (Thermo Fisher) were employed to quantify the expression levels of miR-139-5p, with U6 serving as an internal control. For the quantification of mRNA and circRNA, PrimeScript RT Master Mix was used to synthesize CDNA samples. Then, qRT-PCR was performed using $100 \mathrm{ng}$ CDNA samples and SYBR Premix Ex Taq II (Takara, Japan) with GAPDH serving as an internal control. The expression levels of mRNA, miRNA, and circRNAs were figured out by the $2^{-\Delta \Delta C T}$ method.

\section{Tumor xenografts in nude mice}

Before the in vivo study, the EXOs were isolated from the culture medium of normally cultured M2-polarized macrophages, EXOs that overexpressed hsa_circ_0001610 (EXO ${ }^{\text {circ_00001610 }}$ ) and negative control of EXO'irc_0001610 (EXO ${ }^{\text {Vector }}$ ) were isolated from the culture medium of M2-polarized macrophages transfected with circ_0001610 and vector, and EXOs that silenced hsa_circ_0001610 (EXO $\left.\mathrm{O}^{\text {sh-circRNA }}\right)$ were isolated from the culture medium of $\mathrm{M} 2$-polarized macrophages transfected with sh-circRNA respectively. Also, $5 \times 10^{6}$ Ishikawa or HEC-1B cells were suspended in a $50 \mu \mathrm{l}$ mixture of DMEM and Matrigel (volume ratio $=1: 1$ ) and subcutaneously injected into the BALB/c nude mice (4-week-old; Beijing Charles River Experimental Animal Technology Co., Ltd, China). When tumors grew to $100 \mathrm{~mm}^{3}$, mice were allocated to five groups: Control $(n=6)$, EXO $(n=$ $6)$, EXO ${ }^{\text {circ_co001610 }}(n=6), \operatorname{EXO}^{\text {Vector }}(n=6)$, and $\operatorname{EXO}^{\text {sh-circRNA }}(n=6)$. In the control group, the tumors were locally irradiated with $10 \times 2.5 \mathrm{~Gy}$ for 5 days. In the EXO/EXO Vector/EXO'irc_0001610/ EXO EXO/EXO Vector/EXO the tumor every 2 days, and the tumors were locally irradiated with $10 \times$ $2.5 \mathrm{~Gy}$ for 5 days. Tumor volumes were measured every week after the beginning of local irradiation. Four weeks after the beginning of local irradiation, mice were sacrificed and the xenotransplanted tumors were collected. All animal experiments were approved by the Committee on the Ethics of The First Affiliated Hospital of Zhengzhou University.

\section{Immunohistochemical staining}

Tumor tissues were prepared into 4- $\mu$ m-thick sections, followed by dewaxing and re-watering. Then, sections were immersed in $3 \%$ hydrogen peroxide. Ten minutes later, sections were orderly incubated with a blocking solution and anti-cyclin B1/Ki-67 antibody (Abcam). On the next day, sections were reacted with a secondary antibody and then counterstained with hematoxylin. A BX63 fluorescence microscope (Olympus Life Science) was used to photograph the stained sections.

\section{Statistical analysis}

GraphPad Prism 7.0 (GraphPad, USA) was used to perform statistical analyses. The results are expressed as the means \pm standard deviations. The statistical significance of the differences between the two experimental groups was evaluated using a Student's $t$ test, and $p<0.05$ was considered statistically significant.

\section{RESULTS}

Macrophages are enriched in the cancerous tissues of patients with EC and characterized by the M2-polarized phenotype

To evaluate the distribution of macrophages in the human EC specimens, the expressions of $\mathrm{CD} 11 \mathrm{~b}$ and CD68, two macrophage markers [21], were measured. Flow cytometric quantification showed a higher density of $\mathrm{CD}_{11} \mathrm{~b}^{+} \mathrm{CD}^{+} 8^{+}$macrophages in $\mathrm{CD}_{4} 5^{+}$immune cells in EC specimens than in normal endometrial specimens (Fig. 1A). Through analyzing the expression of CD206, a specific surface marker of M2-polarized macrophages [22], we found that the proportion of $\mathrm{CD} 206^{+}$cells in EC specimens was $10.7 \%$, but the proportion of $\mathrm{CD} 206^{+}$cells in normal endometrial specimens was too low to be detected (Fig. 1A). TAMs in EC specimens were verified as an M2-like phenotype, characterized by an increased proportion of $\mathrm{CD}_{11 \mathrm{~b}^{+}} \mathrm{CD} 206^{+}$cells (Fig. 1B). Meanwhile, elevations in mRNA levels of M2 markers (IL-10, Arg-1, and TGF- $\beta$ [23]) and reductions in mRNA levels of M1 markers (TNF-a, IL-6, and IL-12 [24]) were observed in TAMs in EC specimens compared with NTMs in normal endometrial specimens (Fig. 1C), further confirming that the TAMs in EC specimens were primarily macrophage subpopulation with an $M 2$ phenotype. The results of immunofluorescent staining also demonstrated that TAMs in EC specimens were F4/80 positive and CD206 positive while NTMs in normal endometrial specimens were $F 4 / 80$ positive and CD206 negative (Fig. 1D).

\section{M2-polarized macrophage-derived EXOs accounted for weakening the radiosensitivity of EC cells}

To assess the functional biology of TAMs on EC cells in vitro, M2polarized macrophages were generated from THP-1 cells, and the influence of M2-polarized macrophages on EC cells' viability, proliferation, invasion, and radiosensitivity was examined. As shown in Fig. 2A, M2-polarized macrophages expressed higher expression levels of CD11b and CD206. The mRNA levels of IL-10, Arg-1, and TGF- $\beta$ in M2-polarized macrophages were also 
A

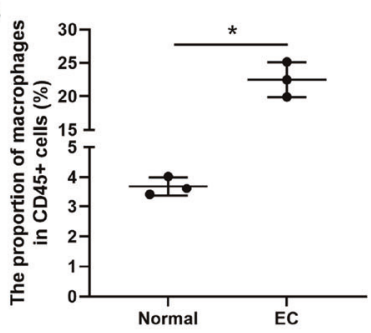

B
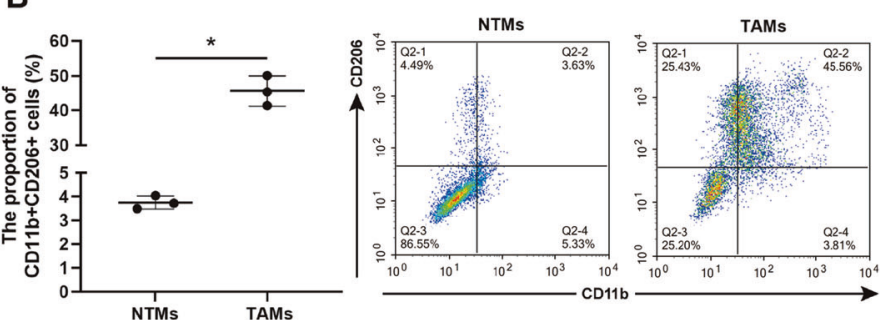

EC

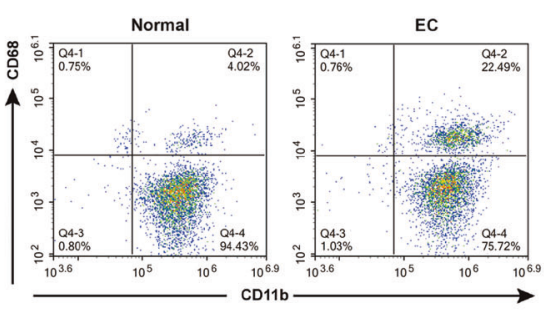

EC

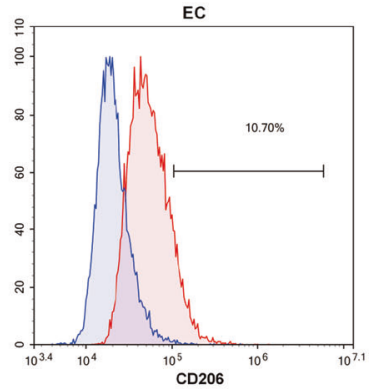

$\mathrm{C}_{12}$

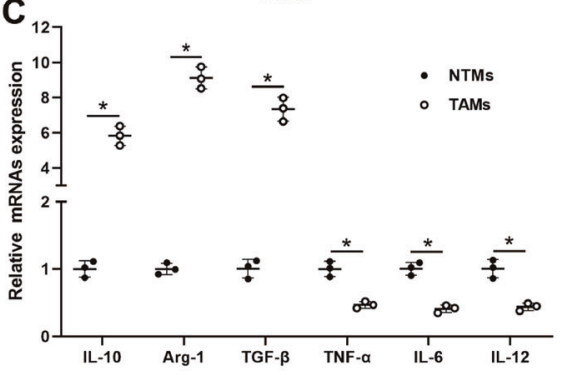

D

DAPI

F4/80
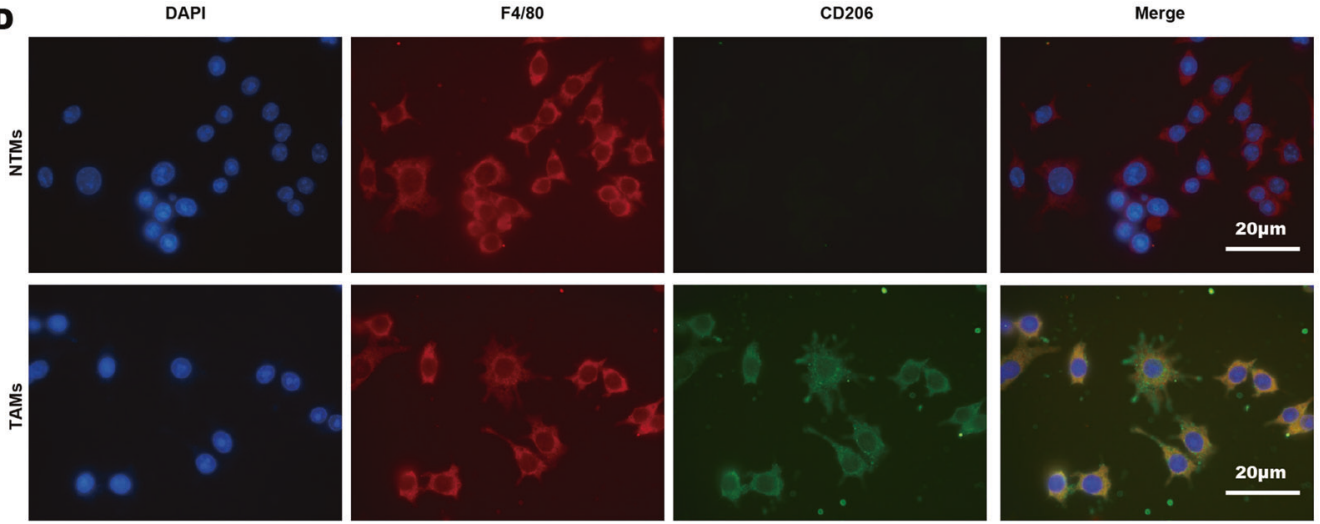

Fig. 1 Accumulation of M2-like TAMs in cancerous tissues of patients with EC. A Flow cytometry analysis and quantification of macrophages in human EC specimens $(n=3)$ and normal endometrial specimens $(n=3)$. Left: $\mathrm{CD}^{+} 5^{+}$cells were gated and then analyzed the CD11 $\mathrm{b}^{+} \mathrm{CD}^{+} 8^{+}$cells. Right: The proportion of CD206 ${ }^{+}$cells in human EC specimens. B Flow cytometry analysis and quantification of CD11 ${ }^{+}$ $\mathrm{CD}^{206^{+}}$macrophages in tissue-resident macrophages (NTMs, $\left.n=3\right)$ and tumor-associated macrophages (TAMs, $n=3$ ). C qRT-PCR analysis of IL-10, arginase-1 (Arg-1), transforming growth factor-beta (TGF- $\beta$ ), tumor necrosis factor-alpha (TNF- $\alpha$ ), IL-6, and IL-12 mRNA levels in NTMs $(n=3)$ and TAMs $(n=3)$. * $p<0.05$. D The representative images of immunofluorescent staining for F4/80 (red) and CD206 (green) performed on TAMs and NTMs. Nuclei were counterstained with DAPI (blue). Scale bar $=20 \mu \mathrm{m}$.

upregulated compared to those in nonpolarized $\mathrm{M} 0$ macrophages (Fig. 2B). Compared with monocultured Ishikawa and Ishikawa cells cocultured with M0 macrophages, Ishikawa cocultured with M2-polarized macrophages exhibited higher viability, increased colony numbers, and enhanced invasive ability (Supplementary Fig. $1 \mathrm{~A}-\mathrm{C})$, indicating that $\mathrm{M} 2$-polarized macrophages promoted the malignant phenotype of EC cells.

Further, the effect of TAMs on the radiosensitivity of EC cells was evaluated. Following treatment with $4 \mathrm{~Gy}$ irradiation, EC cell lines (Ishikawa and HEC-1B) cocultured with M2-polarized macrophages presented higher cell viability, increased colony numbers, reinforced invasive ability, and decreased cell apoptosis compared with monocultured EC cell lines (Fig. 2C-F) and EC cell line cocultured with M0 macrophages (Supplementary Fig. 1D-F). Meanwhile, the cell cycle distribution also showed that the rate of G2/M-arrested cells was lessened in EC cell lines (Ishikawa and HEC-1B) cocultured with M2-polarized macrophages than in monocultured EC cell lines after $4 \mathrm{~Gy}$ irradiation treatment (Fig. $2 \mathrm{G}$ ). These data suggested that M2-polarized macrophages weakened the radiosensitivity of EC cells.

Given that emerging evidence confirms that EXOs derived from TAMs play a central role in the communication between tumor cells and TAMs $[25,26]$, we then explored whether TAM-derived EXOs mediated the radiosensitivity of EC cells. The conditioned medium (CM) and conditioned medium deleted EXO ( $\left.C M^{\text {de-EXO }}\right)$ were harvested from the M2-polarized macrophage culture medium (Supplementary Fig. 2). Like M2-polarized macrophages, the CM treatment also upregulated cell viability (Fig. 2C), proliferation (Fig. 2D), and invasion (Fig. 2E), and downregulated cell apoptosis (Fig. 2F) and G2/M cell cycle arrest (Fig. 2G) in EC cell lines (Ishikawa and HEC-1B) exposed to $4 \mathrm{~Gy}$ irradiation. In contrast, EC cell lines (Ishikawa and HEC-1B) incubated with $\mathrm{CM}^{\text {de-EXO }}$ presented lower cell viability, suppressed capacities of proliferation and invasion, promoted cell apoptosis, and induced G2/M cell cycle arrest compared with EC cell lines incubated with $\mathrm{CM}$ after $4 \mathrm{~Gy}$ irradiation treatment (Fig. $2 \mathrm{C}-\mathrm{G}$ ). Collectively, the above data indicated that EXOs derived from M2-polarized macrophages attenuated the radiosensitivity of EC cells.

Exosomal hsa_circ_0001610 mediated the weakening effect of M2-polarized macrophage-derived EXOs on the radiosensitivity of EC cells

Following, the mechanism by which EXOs derived from M2polarized macrophages conferred radioresistance of EC cells 
A
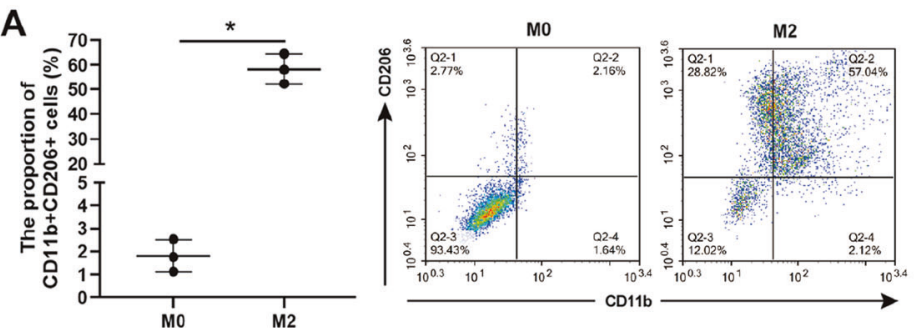

C
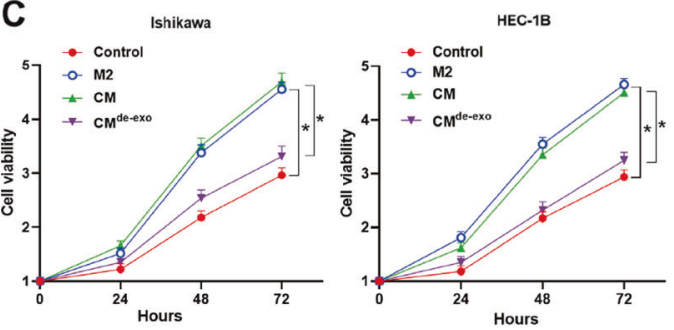

D
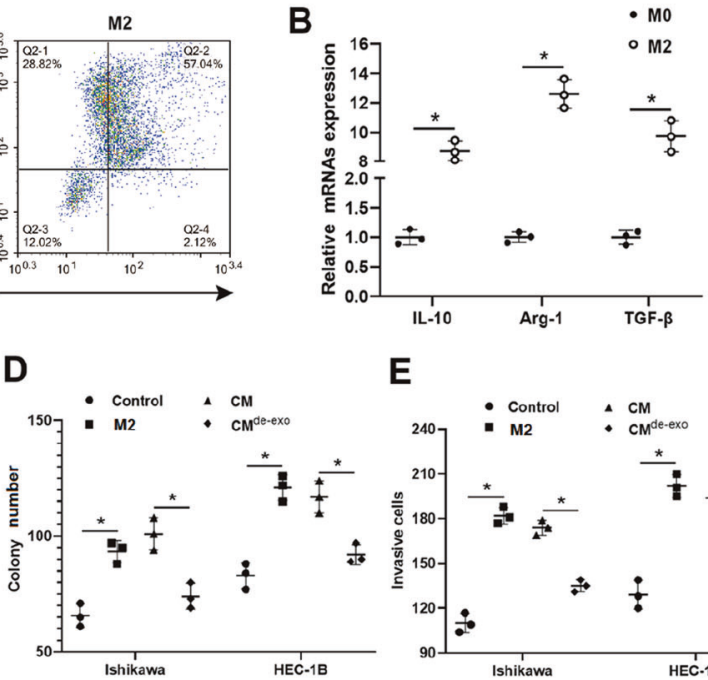

E
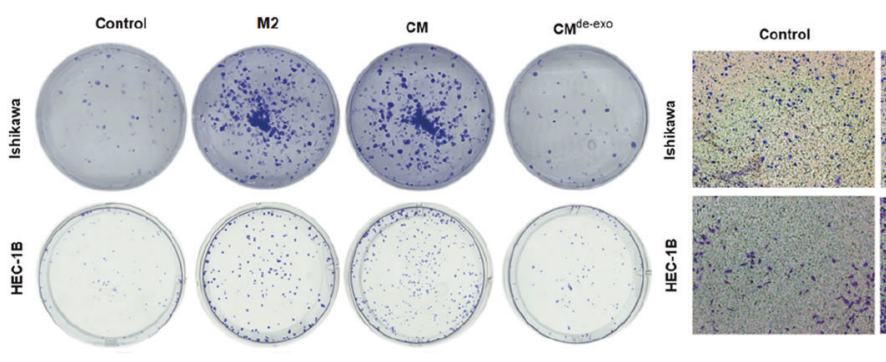

M2
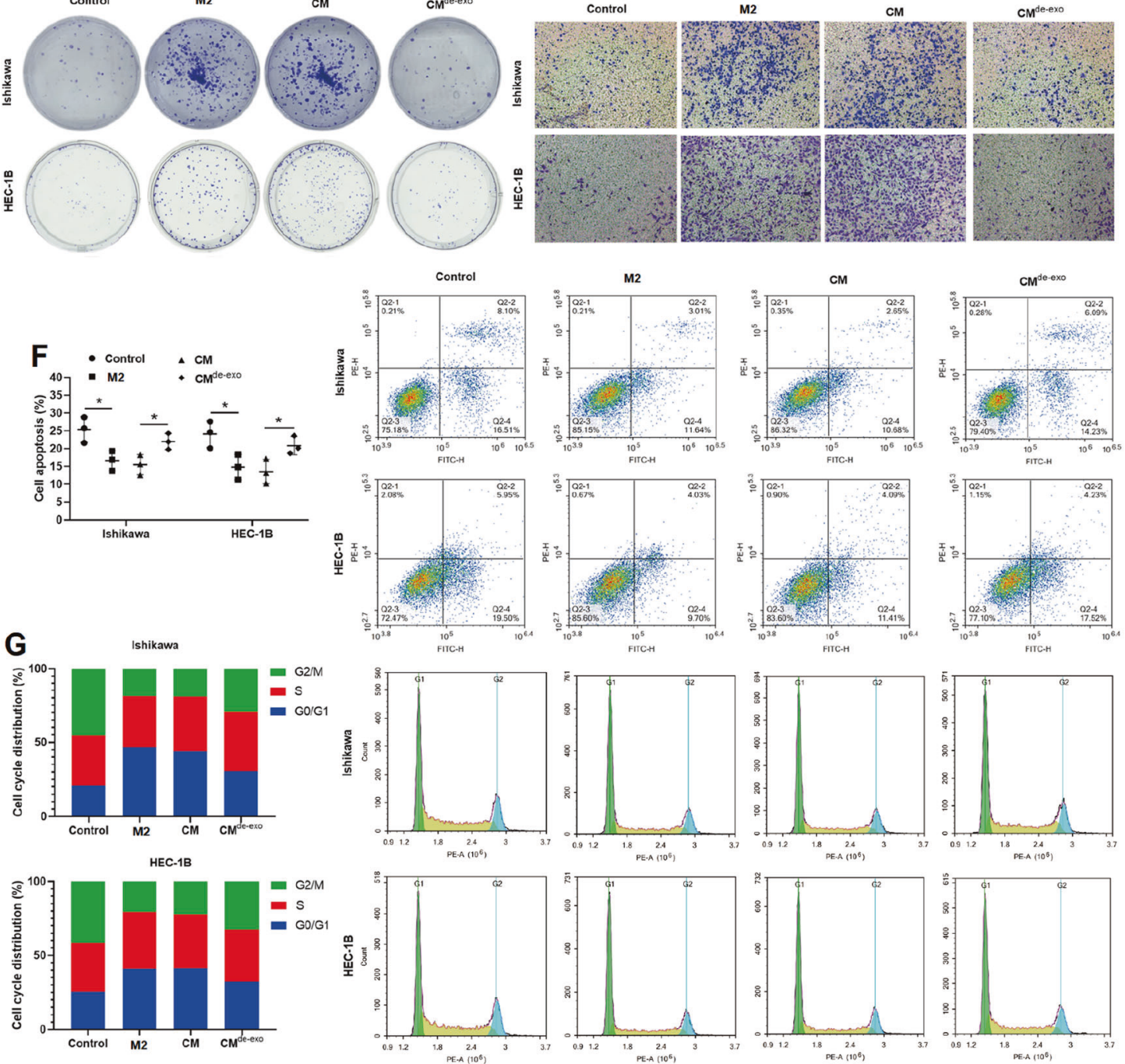

Fig. 2 M2-polarized macrophage-derived exosomes weakened the radiosensitivity of EC cells. A Flow cytometry analysis and quantification of $\mathrm{CD} 11 \mathrm{~b}^{+}$CD206 ${ }^{+}$cells and B qRT-PCR analysis of IL-10, Arg-1, and TGF- $\beta$ mRNA levels were performed on nonpolarized M0/ M2-polarized macrophages generated from THP-1 cells. C-G The conditioned medium (CM) and conditioned medium deleted exosomes $\left(\mathrm{CM}^{\mathrm{de}-\mathrm{EXO}}\right)$ were harvested from the M2-polarized macrophages culture medium. EC cell lines (Ishikawa and HEC-1B) were monocultured or cocultured with M2-polarized macrophages/CM/CM ${ }^{\text {de-EXO }}$ and then treated with $4 \mathrm{~Gy}$ irradiation. C Cell viability was measured by 3-(4,5dimethylthiazol-2-yl)-2,5-diphenyltetrazolium bromide (MTT) assay. D Cell proliferation was measured by colony formation assay. E Cell invasion was measured by Transwell assay. $\mathbf{F}$ Cell apoptosis was measured by flow cytometry. $\mathbf{G}$ Cell cycle distribution was determined by propidium iodide (PI) staining. ${ }^{*} p<0.05$. 
was investigated. The EXOs were isolated from M2-polarized macrophages by differential centrifugation. TEM imaging analysis showed that purified EXOs morphologically resembled a cupshaped structure and were around $100 \mathrm{~nm}$ in size (Fig. 3A). Western blot analysis showed that purified EXOs were positive for the EXO-specific markers CD63, CD9, and CD81 [27] (Fig. 3A). These data confirmed the success of EXO isolation. Increasing evidence reports that several circRNAs participate in EC progression and that circRNAs encapsulated in EXOs play a vital role in implementing the cellular function of EXOs [28, 29]. To explore whether circRNAs were involved in M2-polarized macrophagederived EXOs-mediated radiosensitivity of EC cells, the following study was conducted. Through a circRNA microarray analysis, we obtained the expression profiles of 10 candidate circRNAs reported to be related to EC development [16, 30-36] in M2polarized macrophage-derived EXOs and found that hsa_circ_0001610 was the most abundant (Fig. 3B). The following results of qRT-PCR also confirmed that hsa_circ_0001610 was abundant in M2-polarized macrophage-derived EXOs rather than EC cell-derived EXOs (Fig. 3B). The RNA-FISH assay showed that hsa_circ_0001610 was localized to the cytoplasm of M2-polarized macrophages and Ishikawa cells (Fig. 3D).

Then, EXOs that silenced hsa_circ_0001610 (EXO ${ }^{\text {sh-circRNA }}$ ), its negative control (EXO ${ }^{\text {shRNA }}$ ), and normal EXOs were prepared (Fig. 3C) and added into the culture mediums of Ishikawa and HEC-1B cells before $4 \mathrm{~Gy}$ irradiation treatment. Compared with monocultured EC cell lines, EC cell lines treated with EXOs presented increased cell viability (Fig. 3E), promoted cell proliferation (Fig. $3 F$ ), enhanced cell invasion (Fig. 3G), suppressed cell apoptosis (Fig. 3H), and lessened rate of G2/M-arrested cells (Fig. 3I) after $4 \mathrm{~Gy}$ irradiation treatment. However, the EXO-induced radioresistance of EC cells was partially reversed when silencing hsa_circ_0001610 in M2-polarized macrophage-derived EXOs. As shown in Fig. 3E-l, EXO ${ }^{\text {sh-circRNA }}$, rather than EXO ${ }^{\text {sh-RNA }}$, efficiently reduced cell viability, repressed cell proliferation, weakened cell invasion, reinforced cell apoptosis, and augmented the rate of G2/ $M$-arrested cells in EC cell lines treated with $4 \mathrm{~Gy}$ irradiation. Overall, these data suggest that M2-polarized macrophagederived EXOs reduce the radiosensitivity of EC cells by loading hsa_circ_0001610.

\section{The interplay between hsa_circ_0001610, miR-139-5p, and cyclin B1}

As reported, circRNAs could act as competing endogenous RNAs (ceRNAs), competitively combining with miRNAs to affect the expression levels of target mRNAs, thereby exerting their modulatory effect on radiosensitivity in several types of cancers $[13,14]$. According to the bioinformatics database (Circular RNA Interactome), 55 miRNAs were found to have potential binding sites to hsa_circ_0001610. Of them, 14 miRNA have been reported to be related to EC development (Supplementary Fig. 3). Their expression levels were measured using qRT-PCR in Ishikawa cells transfected with hsa_circ_0001610. As shown in Supplementary Fig. 3, miR-139-5p level demonstrated the most significant change upon hsa_circ_0001610 overexpression. Therefore, miR-139-5p was selected for the following investigation. The potential binding sites between miR-139-5p and hsa_circ_0001610 were shown in Fig. 4A. The results of the dual-luciferase reporter assay showed that the relative luciferase activity of the pmirGLO-circ_0001610 vector, rather than the pmirGLO-mutant-circ_0001610 vector, was significantly decreased by miR-139-5p mimic transfection in HEK293 T cells (Fig. 4B). The results of the RNA pull-down assay showed a sixfold enrichment of hsa_circ_0001610 in the biotin-miR-139$5 \mathrm{p}$-captured fraction compared with the control RNA-captured fraction in EC cell lines (Fig. 4C). Meanwhile, miR-139-5p expression was downregulated by hsa_circ_0001610 overexpression whereas upregulated by hsa_circ- 0001610 knockdown in EC cell lines (Fig. 4D). These data confirmed that hsa_circ_0001610 was directly bound to miR-139-5p and negatively regulated its expression in EC cell lines.

Using a bioinformatics database (TargetScan), we found that cyclin B1, a vital pusher of radioresistance in several types of cancer by regulating the cell cycle [37-39], was the potential target of miR-139-5p and the potential binding sites between CCNB1 (the encoding gene of cyclin B1) and miR-139-5p are shown in Fig. 4E. A distinct reduction in the relative luciferase activity of pmirGLO-CCNB1 3'-UTR WT vector in miR-139-5p mimic-transfected HEK-293T cells indicated the interplay between miR-139-5p and CCNB1 (Fig. 4E). Furthermore, the results of qRTPCR and Western blot demonstrated that both mRNA and protein levels of cyclin B1 were lessened by miR-139-5p mimic transfection in EC cell lines (Fig. 4F, G), which suggested that cyclin B1 expression was negatively regulated by miR-139-5p.

\section{Hsa_circ_0001610 weakened the radiosensitivity of EC cells by} suppressing miR-139-5p expression

Next, we explored whether hsa_circ_0001610 reduced the radiosensitivity of EC cells by modulating miR-139-5p. Ishikawa and HEC-1B cells were transfected with vector, hsa_circ_0001610, hsa_circ_0001610+miR-NC, or hsa_circ_0001610+miR-139-5p mimic, followed by exposure to $4 \mathrm{~Gy}$ irradiation. The hsa_circ_0001610 transfection overtly promoted circ_0001610 level (Fig. 5A), decreased miR-139-5p level (Fig. 5B), and increased cyclin B1 level (Fig. 5C, D) in EC cell lines treated with 4Gy irradiation. The miR-139-5p mimic transfection efficiently reversed the regulatory effect of hsa_circ_0001610 on the expressions of miR-139-5p and cyclin B1, which was manifested in upregulating miR-139-5p level (Fig. 5B) and reducing cyclin B1 level (Fig. 5C, D) in EC cell lines treated with 4 Gy irradiation + hsa_circ_0001610 + miR-139-5p mimic. Furthermore, in EC cell lines treated with $4 \mathrm{~Gy}$ irradiation, hsa_circ_0001610 overexpression elevated cell viability (Fig. 5E), boosted cell proliferation (Fig. 5F), enhanced cell invasion (Fig. 5G), suppressed cell apoptosis (Fig. 5H), and reduced G2/M cell cycle arrest (Fig. 5I). In contrast, miR-139-5p overexpression reenhanced the radiosensitivity of EC cells in the presence of hsa_circ_0001610, which was manifested in the downregulations of cell viability (Fig. 5E), proliferation (Fig. 5F), invasion (Fig. 5G), the upregulations of cell apoptosis (Fig. $5 \mathrm{H}$ ), and the rate of G2/Marrested cells (Fig. 5I) in EC cell lines treated with 4 Gy irradiation + hsa_circ_0001610 + miR-139-5p mimic.

\section{Exosomal hsa_circ_0001610 weakened the radiosensitivity of EC cells in vivo}

Then, the role of exosomal hsa_circ_0001610 in the radiosensitivity of EC cells was investigated in vivo. Ishikawa or HEC$1 \mathrm{~B}$ cells were subcutaneously injected into nude mice to establish the tumor xenograft model. When tumors grew to $100 \mathrm{~mm}^{3}$, mice were treated with irradiation, irradiation + EXO, irradiation +

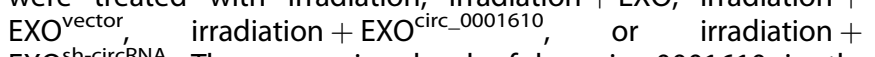
EXO $^{\text {sh-circkNA }}$. The expression level of hsa_circ_0001610 in the tumors, measured by GRT-PCR, confirmed that the injection of

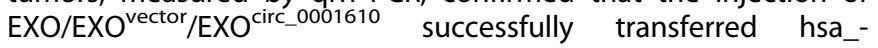
circ_0001610 to the xenotransplanted tumors of nude mice, among which the mice that received EXO'irc_0001610 injection presented the most abundant hsa_circ_0001610 (Fig. 6C). As shown in Fig. 6A, B, the tumor volume and tumor weight of nude mice injected with EC cells + EXO was increased compared with nude mice only injected with EC cells. In addition, the expression level of miR-139-5p was decreased (Fig. 6D), and the protein levels of cyclin B1 and Ki-67 (a proliferation marker [40]) (Fig. 6E) were promoted in the tumors of nude mice injected with EC cells + EXO compared to the tumors of nude mice injected with EC cells. However, the effects of EXO on tumor volume, tumor weight, and the expression levels of miR-139-5p, cyclin B1, and Ki-67 were removed when silencing hsa_circ_0001610 (EXO ${ }^{\text {sh-circRNA }}$ ) (Fig. 6A-D). Compared with EXO ${ }^{\text {vector }}$, the administration of 


\section{A}

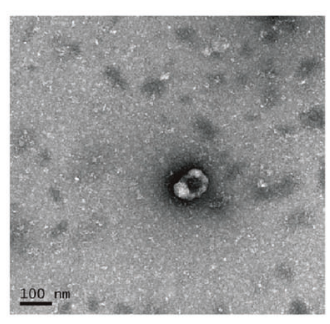

C

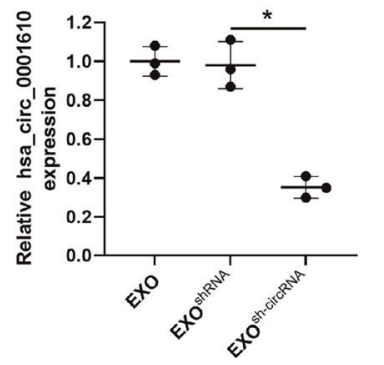

E

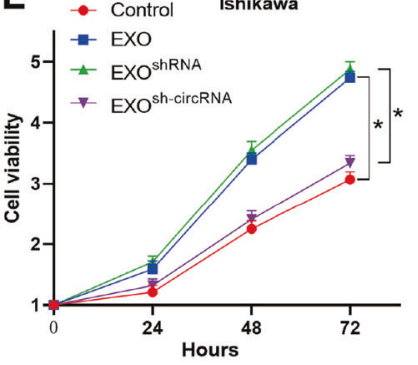

$\rightarrow$ Control HEC-1B
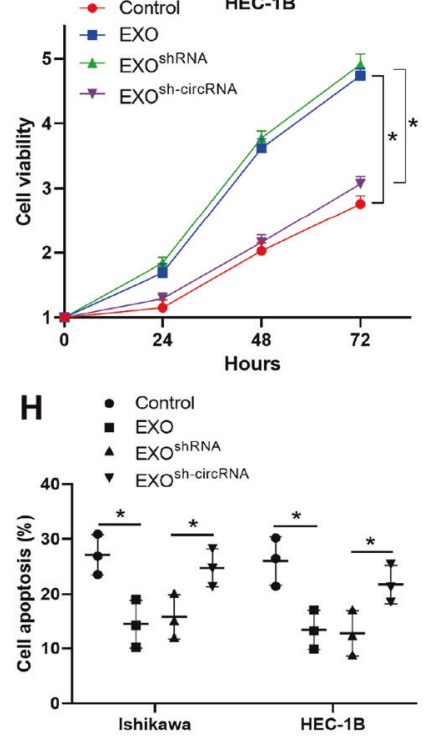

D
B
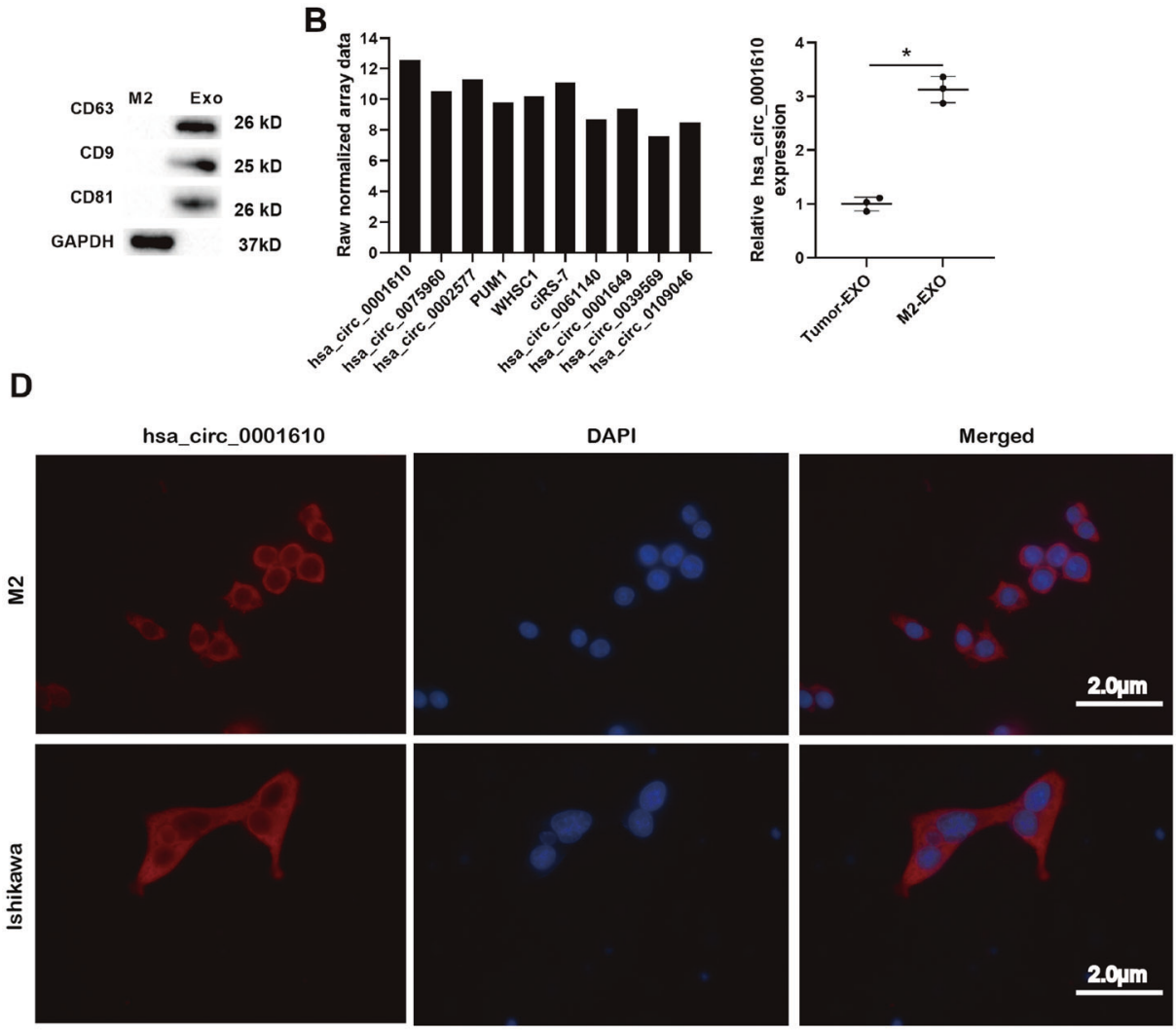

F - Control

- EXo

- Exo shrna

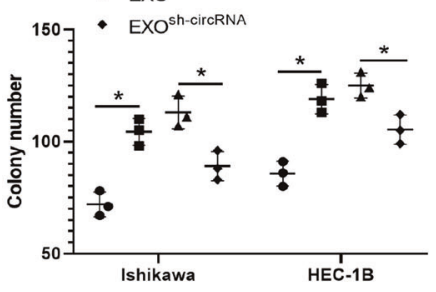

G

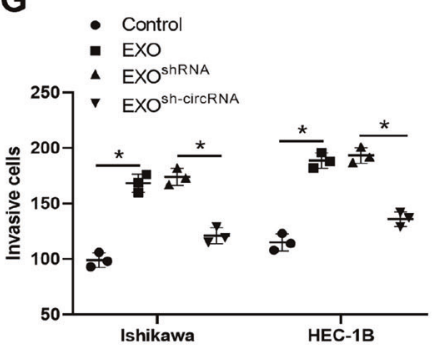

I

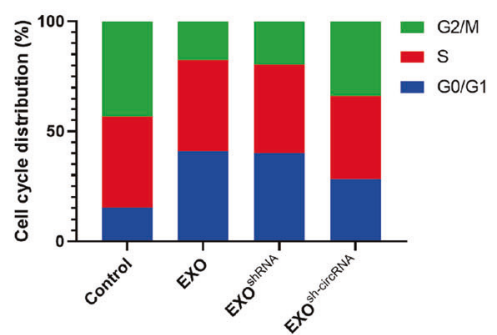

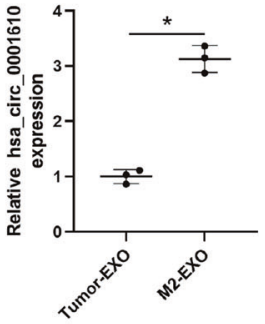

2.04

Fig. 3 Exosomal hsa_circ_0001610 mediated the weakening effect of M2-polarized macrophage-derived EXOs on the radiosensitivity of EC cells. A Representative transmission electron microscopy image of M2-polarized macrophage-derived EXOs (Scale bar $=100 \mathrm{~nm})$. B Left: the expression profiles of ten candidate circRNAs in M2-polarized macrophage-derived EXOs were generated by a circRNA microarray analysis. Right: the expression level of hsa_circ_0001610 in EC cell-derived EXOs and M2-polarized macrophage-derived EXOs was measured by qRTPCR. C The expression level of hsa_circ_0001610 in M2-polarized macrophage-derived EXOs (EXO), EXOs that silenced hsa_circ_0001610 $\left(E O^{\text {sh-circRNA }}\right)$, and the negative control of EXO ${ }^{\text {sh-circRNA }}\left(\right.$ EXO $\left.^{\text {sh-RNA }}\right)$ was measured by qRT-PCR. D The cellular localization of hsa_circ_0001610 in M2-polarized macrophages and Ishikawa cells (Scale bar $=2.0 \mu \mathrm{m}$ ). E-I EC cell lines (Ishikawa and HEC-1B) were normally cultured (Control) or treated with EXO/EXO ${ }^{\text {sh-RNA }} / \mathrm{EXO}^{\text {sh-circRNA }}$, and then treated with $4 \mathrm{~Gy}$ irradiation. Cell $\mathbf{E}$ viability, $\mathbf{F}$ proliferation, $\mathbf{G}$ invasion, $\mathbf{H}$ apoptosis, and I cell cycle were measured. ${ }^{*} p<0.05$. 
A

hsa_circ_0001610 5' UAGAGAAAUGCCAUGACUGUAGU 3' |||||| hsa-miR-139-5p 3' GACCUCUGUGCACGUGACAUCU 5'
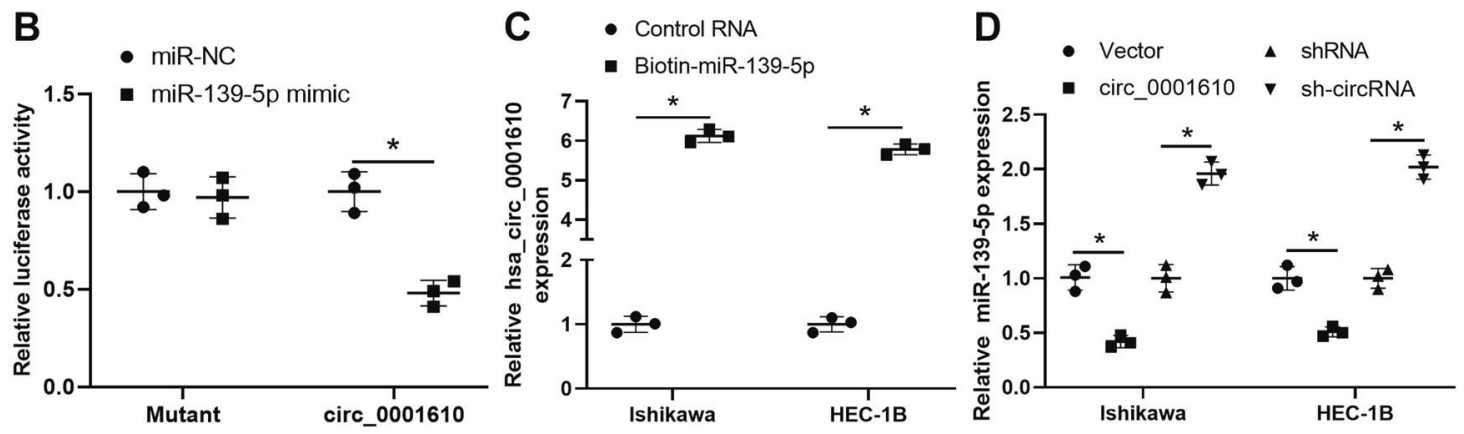

E

CCNB1 5' AUgugguUACUUCCUACUgUAGG 3' |||||| $\mid$ 'miR-139-5p 3' GACCUCUGUGCACGUGACAUCU 5'
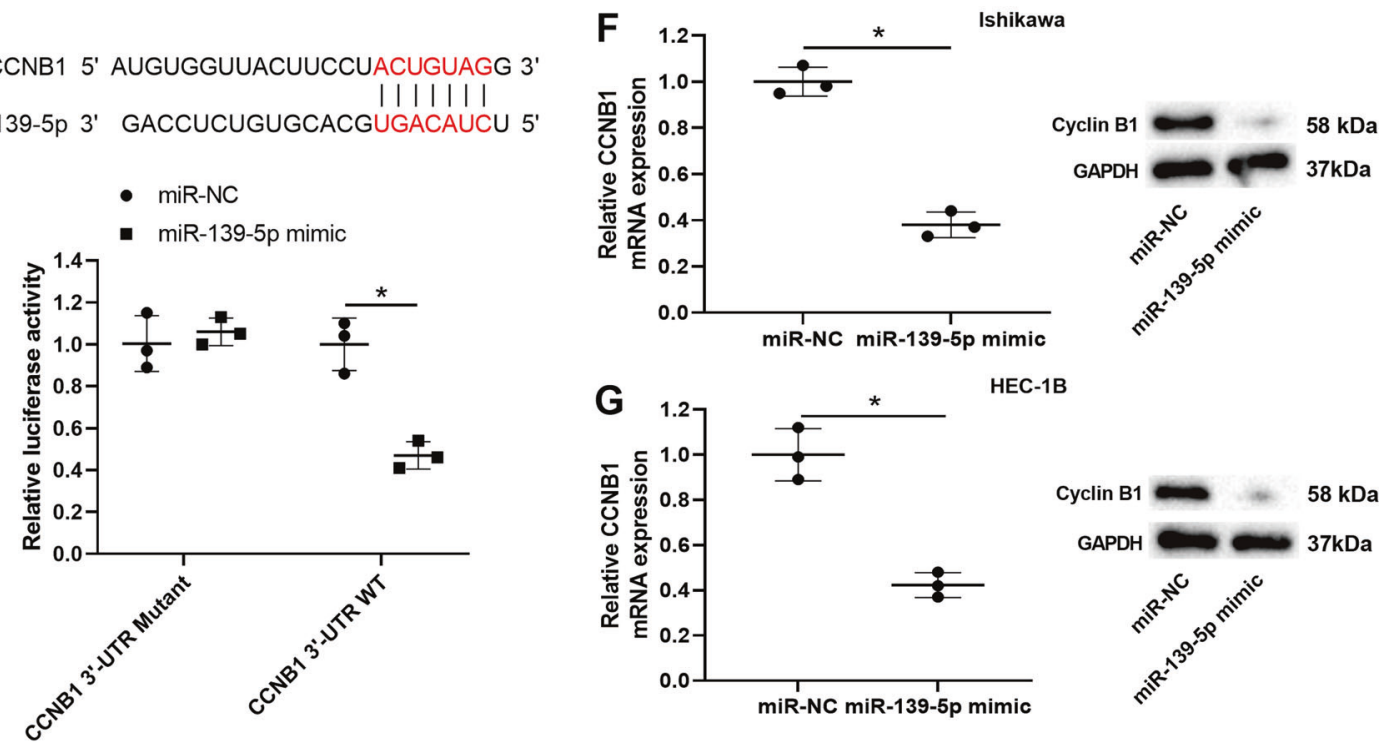

EC-1B

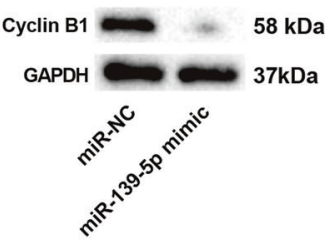

Fig. 4 The interplay between hsa_circ_0001610, microRNA (miR)-139-5p, and cyclin B1. A The potential binding sites between hsa_circ_0001610 and miR-139-5p. B Dual-luciferase reporter assay using HEK-293T cells co-transfected with pmirGLO-circ_0001610 vector/ pmirGLO-mutant-circ_0001610 vector and miR-139-5p mimic/the negative control of miR-139-5p mimic (miR-NC). C qRT-PCR analysis of hsa circ 0001610 level in the streptavidin captured fractions from the Ishikawa and HEC-1B cell lysates after incubation with biotinylated miR139-5p (biotin-miR-139-5p) or control RNA. D qRT-PCR analysis of miR-139-5p in the Ishikawa and HEC-1B cells after transfection with circ_0001610/the negative control of circ_0001610 (vector)/sh-circRNA/shRNA. E Upper: the potential binding sites between CCNB1 (the encoding gene of cyclin B1) and miR-139-5p. Below: dual-luciferase reporter assay using HEK-293T cells co-transfected with pmirGLO-CCNB1 $3^{\prime}$-UTR mutant vector/ pmirGLO-CCNB1 3'-UTR wild type (WT) vector and miR-139-5p mimic/ miR-NC. The mRNA and protein levels of cyclin B1 were measured in $\mathbf{F}$ Ishikawa and $\mathbf{G}$ HEC-1B cells after transfection with miR-139-5p mimic/miR-NC. ${ }^{*} p<0.05$.

EXO ${ }^{\text {circ }} 0001610$ resulted in increased tumor volume and weight (Fig. 6A, B), a lower miR-139-5p level (Fig. 6D), and higher protein levels of cyclin B1 and Ki-67 (Fig. 6E) in the tumors of nude mice injected with EC cells. Moreover, EXO facilitated the EMT in the tumor xenograft model mice after irradiation, manifesting as the upregulations of ZEB1, N-cadherin, $\beta$-catenin, and vimentin, and the downregulation of E-cadherin. The effect of EXO on EMT was enhanced by circ_0001610 overexpression (EXO'circ_0001610) while removed by circ_0001610 knockdown (EXO $\left.{ }^{\text {sh-circRNA }}\right)$. These data suggested that exosomal hsa_circ_0001610 contributed to the EXO-weakened radiosensitivity of EC cells.

\section{DISCUSSION}

In recent years, researchers have come to appreciate that tumors comprise not only tumor cells but also TME. TAMs are the vital components of TME, which promote tumor formation, development, recurrence, and metastasis [41, 42]. Radiotherapy triggers cell death by inducing double-stranded DNA damage. However, under radiotherapy, TAMs promote the radioresistance of cells by inducing immunosuppression, ultimately leading to radiotherapy failure $[43,44]$. Therefore, targeting TAMs may be a novel direction for enhancing radiotherapy potency. Here, we identified a mechanism by which TAMs communicate with EC cells to reduce their radiosensitivity. Our data demonstrated that TAMderived EXOs transferred hsa_circ_0001610 to EC cells, and the overexpressed hsa_circ_0001610 in EC cells released cyclin B1 expression through adsorbing miR-139-5p, thereby weakening the radiosensitivity of EC cells.

Macrophages are extremely plastic cells classified as either M1 or M2 phenotypes [45]. M1-polarized macrophages participate in innate host defense and exhibit an antitumor effect, while M2polarized macrophages participate in humoral immunity and exhibit a pro-tumor effect [46]. Increasing evidence confirms that 

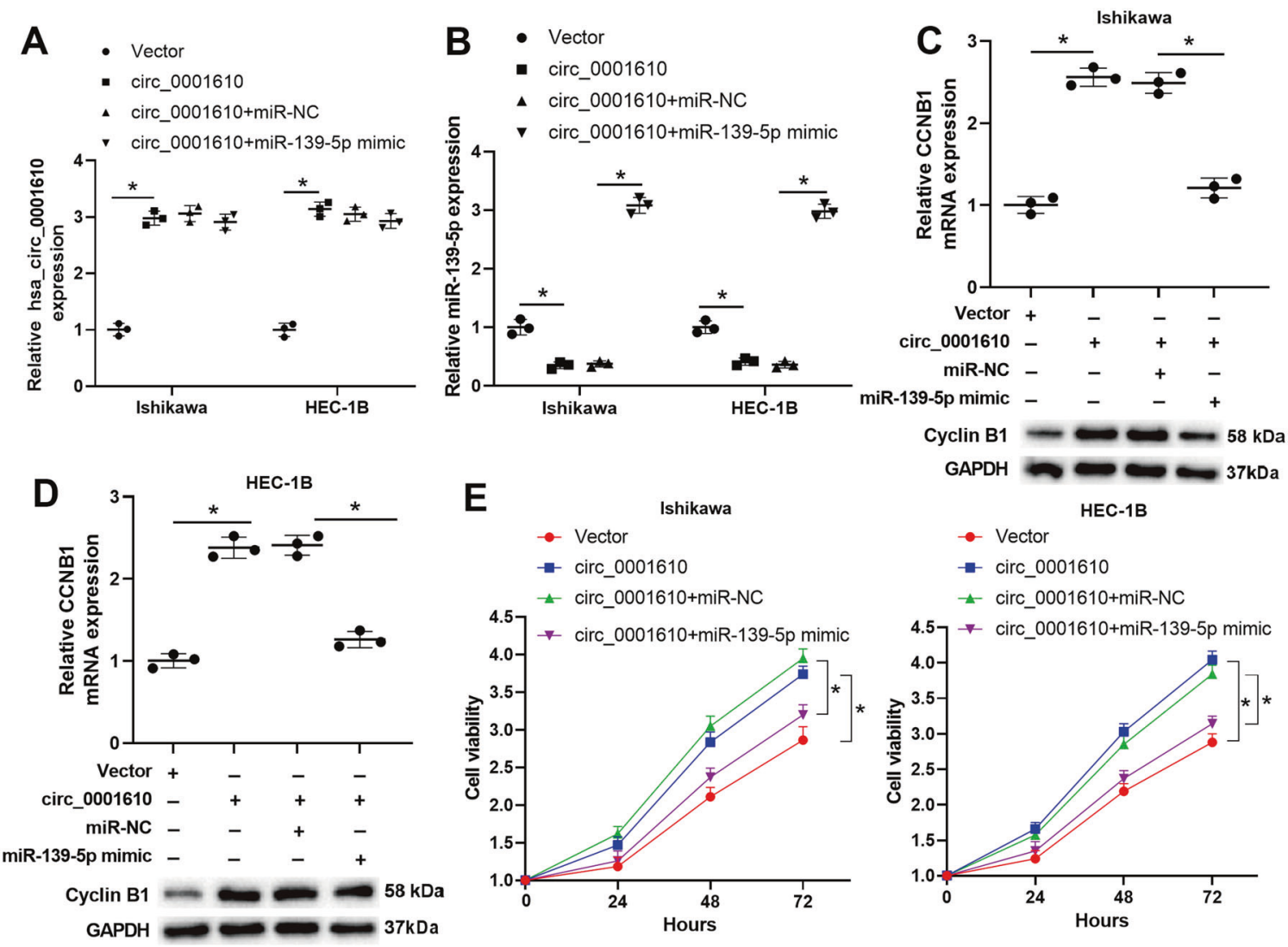

GAPDH

$\rightarrow$ Vector

- circ_0001610

\ circ 0001610+miR-NC
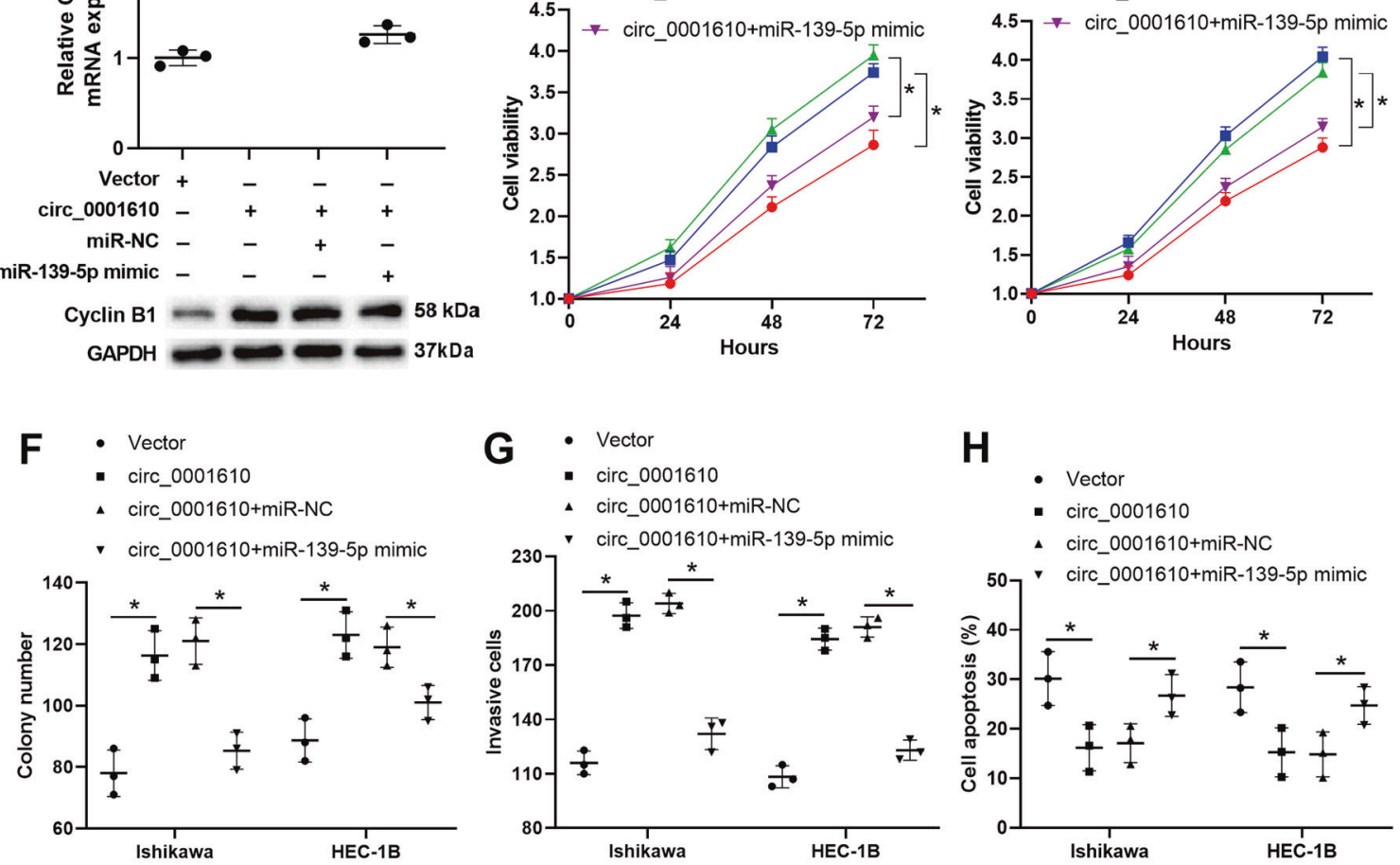
G - Vector
- circ_0001610
- circ_0001610+miR-NC

- circ_0001610+miR-139-5p mimic
H
- Vector
circ 0001610
- circ_0001610+miR-NC
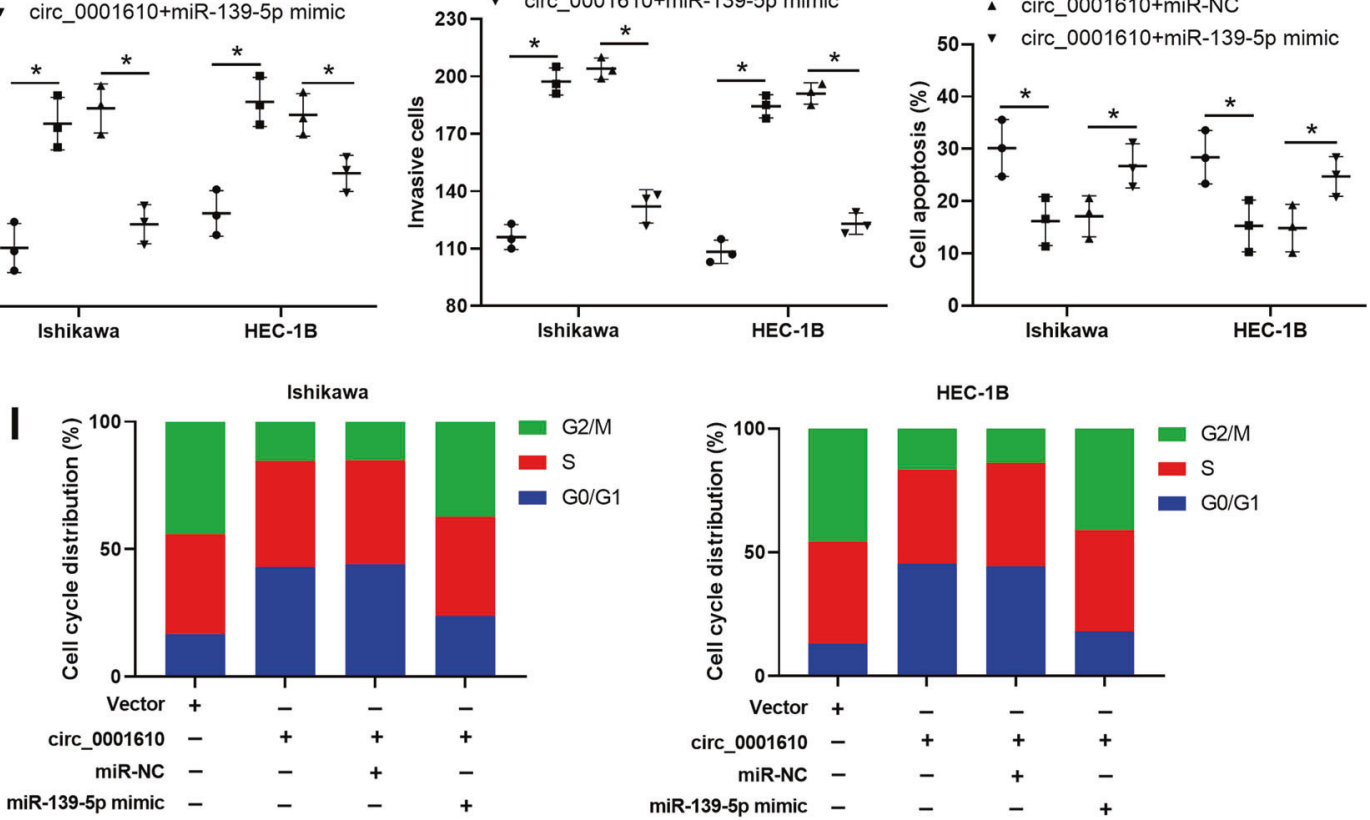

Fig. 5 Hsa circ 0001610 weakened the radiosensitivity of EC cells by suppressing the miR-139-5p expression. Ishikawa and HEC-1B cells were transfected with vector, hsa_circ_0001610, hsa_circ_0001610 + miR-NC, or hsa_circ_0001610 + miR-139-5p mimic, followed by exposure to $4 \mathrm{~Gy}$ irradiation. The expression levels of $\mathbf{A}$ hsa_circ_0001610 and B miR-139-5p was measured by qRT-PCR. The mRNA and protein levels of cyclin B1 in C Ishikawa and D HEC-1B cells were measured by qRT-PCR and Western blot, respectively. E Cell viability was measured by MTT assay. F Cell proliferation was measured by colony formation assay. G Cell invasion was measured by Transwell assay. $\mathbf{H}$ Cell apoptosis was measured by flow cytometry. I Cell cycle distribution was determined by PI staining. ${ }^{*} p<0.05$. 


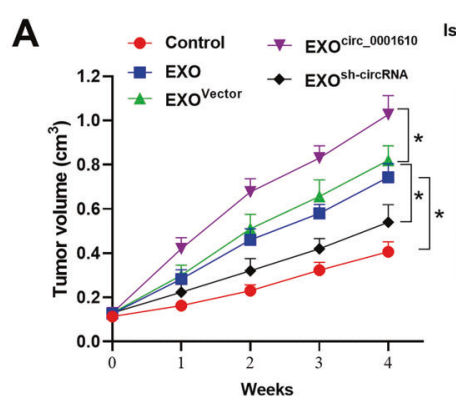

Ishikawa

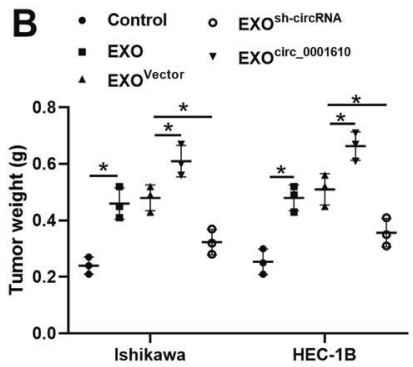

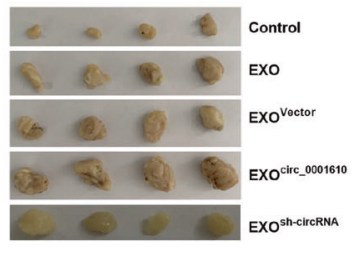

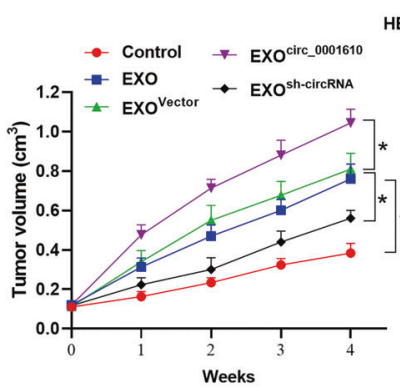

HEC-1B

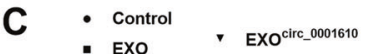

- EXO
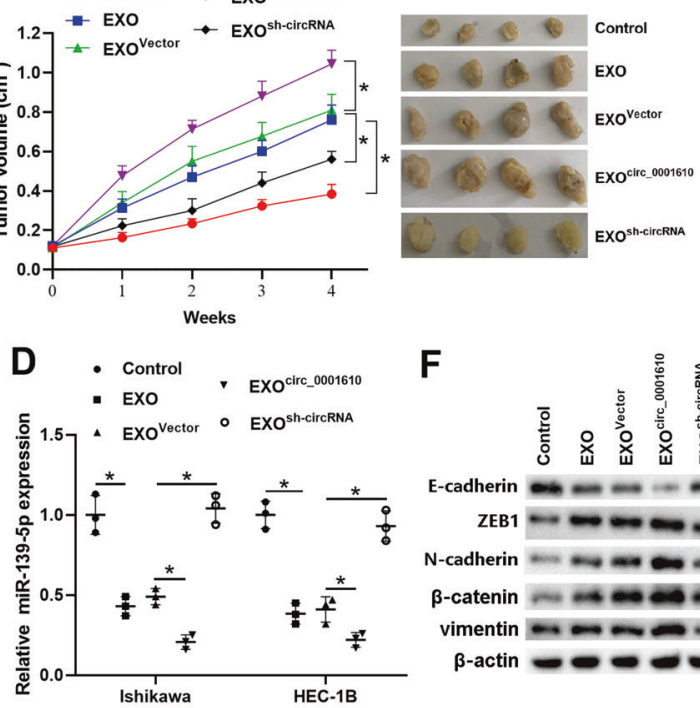

$\mathbf{F}$
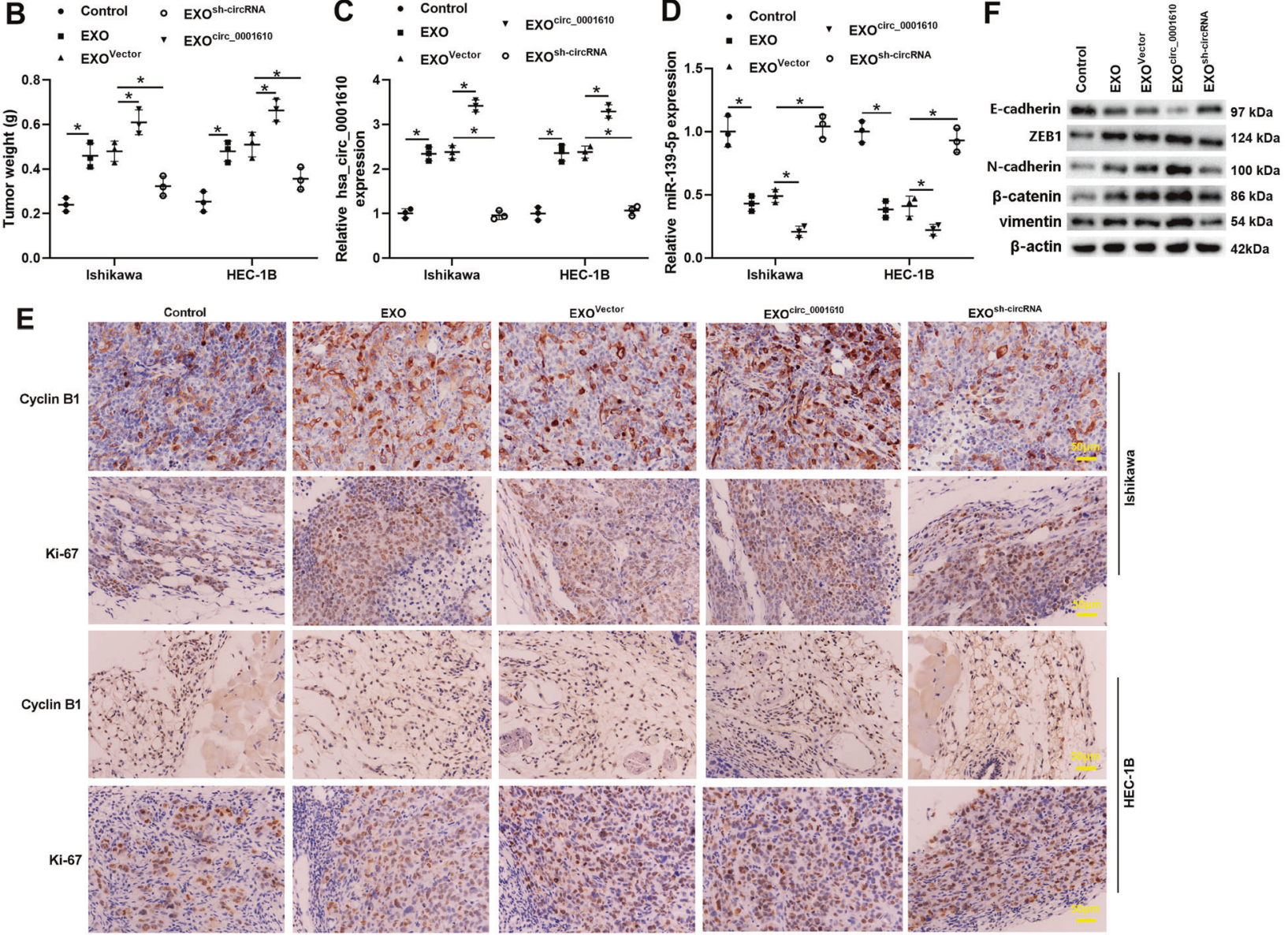

Fig. 6 Exosomal hsa_circ_0001610 weakened the radiosensitivity of EC cells in vivo. Ishikawa or HEC-1B cells were subcutaneously injected into the nude mice to establish the tumor xenograft model. When tumors grew to $100 \mathrm{~mm}^{3}$, mice were treated with irradiation (Control group; $n=6)$, irradiation + EXO (EXO group; $n=6)$, irradiation + EXOs that overexpressed hsa_circ_0001610 $\left(\right.$ EXO $\left.^{\text {circ_0001610 }} ; n=6\right)$, irradiation

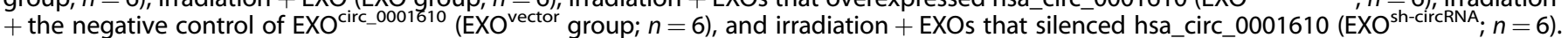
A The tumor volumes were measured every week after the beginning of local irradiation. Four weeks after the beginning of local irradiation, mice were sacrificed, the xenotransplanted tumors were collected and photographed. B Weight of tumors. The expression levels of C hsa_circ_0001610 and D miR-139-5p in tumors were measured. E Representative images of immunohistochemical staining for cyclin B1 and $\mathrm{Ki}-67$ (Scale bar $=50 \mu \mathrm{m})$. F The protein levels of E-cadherin, zinc finger E-box binding homeobox 1 (ZEB1), N-cadherin, $\beta$-catenin, and vimentin. ${ }^{*} p<0.05$.

TAMs are characterized by an M2-polarized phenotype in several types of solid cancer, such as breast cancer [47], gastric cancer [48], and lung cancer [49]. In the present study, an increased TAMs infiltration was observed in the EC specimens relative to normal endometrial specimens, and the following phenotypic analysis revealed that TAMs in EC specimens presented an M2-like phenotype (Fig. 1). Consistent with our data, the research of Gu et al. [7] also reported that TAMs in patients with EC tended to contain a polarized M2 phenotype. Meanwhile, TAMs are proposed to enhance cancer cell resistance against radiotherapy.
Shiao et al. [50] depleted macrophages in the xenograft mammary tumor of mice through colony-stimulating factor 1 (CSF-1) antibody and found that macrophage depletion apparently suppressed tumor growth followed by radiotherapy. Xu et al. [51] reported that compared with irradiation treatment alone, a combination of irradiation and CSF-1 receptor kinase inhibitor more efficiently retard tumor growth in a mouse model of prostate cancer by limiting TAMs recruitment. In line with the previous study, compared with the monocultured EC cell lines, EC cell lines cocultured with M2-polarized macrophages presented 
higher cell viability after irradiation treatment, indicating that M2polarized macrophages decreased the radiosensitivity of EC cells (Fig. 2). What's more, we obtained CM and CM ${ }^{\text {de-EXO }}$ from the M2polarized macrophage culture medium and added them into the culture medium of EC cell lines. The results showed that, like M2polarized macrophages, CM also promoted the viability of EC cell lines treated with irradiation, whereas this ability was removed in the absence of EXOs (Fig. 2), suggesting that M2-polarized macrophages decreased the radiosensitivity of EC cells by secreting EXOs.

As reported, EXOs play a crucial role in cancer progression by transferring various bioactive molecules, such as circRNAs, between cancer cells and TME [52]. Through circRNA microarray and qRT-PCR analysis, we found that hsa_circ_0001610 was abundant in M2-polarized macrophage-derived EXOs (Fig. 3). The experiments that followed showed that knockdown hsa_circ_0001610 in M2-polarized macrophage-derived EXOs removed the promoting effect of $\mathrm{M} 2$-polarized macrophage-derived EXOs on the viability of EC cell lines treated with irradiation, suggesting that exosomal hsa_circ_0001610 reduced the radiosensitivity of EC cells (Fig. 3). This finding was also verified by the accelerative

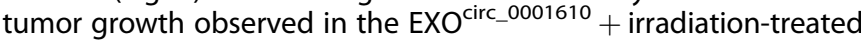
xenograft mouse model (Fig. 6). In the previous study, Ye et al. [36] found the upregulation of hsa_circ_0001610 in the EC tissues of women with grade 3 EC compared with normal endometrial specimens utilizing circRNA sequencing, suggesting the potential role of hsa_circ_0001610 in EC for the first time. Compared to the previous study, the present study not only confirmed the promoting effect of hsa_circ_0001610 on the radioresistance of EC cells but also clarified that the high hsa_circ_0001610 expression in EC tissues may dependent on TAM-derived EXOs transport. In addition, we analyzed the association between hsa_circ_0001610 and the overall survival of EC patients we enrolled and found there was no significant correlation between them ( $p=0.77$, Supplementary Fig. 4 ). Due to the limitation of small sample size, the results in this study may not sufficient to confirm the correlation between hsa_circ_0001610 and the overall survival of EC patients. In future work, we will enroll more EC patients with or without radioresistance to further explore the potential role of hsa_circ_0001610 in prognosis and diagnosis in EC patients with or without radioresistance.

Radiotherapy causes cell apoptosis by damaging DNA double strands and suppressing cell cycle checkpoint activation [53]. G2/ $M$ phase has been reported to be the most radio-sensitive stage of the cell cycle. Several chemotherapeutic agents, such as celecoxib and docetaxel, exert their radiosensitizing effect on tumor cells by inducing G2/M phase arrest [54, 55]. In the present study, an obvious upregulation of G2/M cell cycle arrest was observed in EC cells treated with EXO ${ }^{\text {sh-circRNA }}$ (Fig. 3), hinting that the modulatory effect of hsa_circ_0001610 on the radiosensitivity of EC cells may rely on the cell cycle. Cyclins are critical regulators of the cell cycle. As a key mitotic cyclin, cyclin B1 induces the G2/M phase transition of the cell cycle, thus reducing the radiosensitivity in several types of cancers, such as nasopharyngeal carcinoma, squamous cell carcinoma, and non-small cell lung cancer [56-58]. Through suppressing cyclin B1 expression, proteasome activator complex subunit 3 (PSME3) triggers cell cycle arrest at the G2/M phase and enhances the radiosensitivity of colorectal cancer cells [37]. In EC, the cyclin B1 is overexpressed, and its high expression is closely related to the increased proliferative potential of EC cells [59]. However, whether cyclin B1 contributes to the radioresistance in EC is still unknown. In the present study, using Dual-luciferase reporter assay and RNA pull-down assay, we determined that cyclin B1 was the downstream target of hsa_circ_0001610/miR-139-5p axis and that hsa_circ_0001610 increased cyclin B1 expression via functioning as the ceRNA of miR-139-5p (Figs. 4 and 5). In EC cell lines treated with $4 \mathrm{~Gy}$ irradiation, hsa_circ_0001610 overexpression upregulated cyclin
B1 expression, reinforced cell viability, and reduced the G2/M cell cycle arrest (Fig. 5). However, the miR-139-5p mimic abrogated the promotion effect of hsa_circ_0001610 overexpression on cyclin B1, reduced cell viability, and augmented G2/M cell cycle arrest in circ_0001610 + irradiation-treated EC cell lines (Fig. 5). These data suggest that the weakening effect of circ_0001610 overexpression on radiosensitivity of EC cells is related to its modulatory effect on cyclin B1. The bioinformatics database TargetScan predicted numerous targets of miR-139-5p, and the reason we chose cyclin B1 for this study is that our study focuses on the role of the hsa_circ_0001610/miR-139-5p axis on cell cycle regulation. In the following studies, we will continue to validate other targets of miR-139-5p to more comprehensively elucidate the role of hsa_circ_0001610/miR-139-5p in the progression of EC.

In summary, our study demonstrates that TAM-derived EXOs function as carriers of hsa_circ_0001610, transferring hsa_circ_0001610 to EC cells to adsorb miR-139-5p and release the expression of cyclin B1, thereby weakening the radiosensitivity of EC cells. However, due to objective limitations, we were unable to collect enough samples of EC patients with radioresistance to verify the correlation of this mechanism in clinical samples. Although there are some shortcomings, the present study identifies a new mechanism by which TAMs communicate with EC cells to reduce their radiosensitivity and indicates hsa_circ_0001610 as a potential intervention target of radioresistance in EC.

\section{DATA AVAILABILITY}

The datasets used and/or analyzed during the current study are available from the corresponding author on reasonable request.

\section{REFERENCES}

1. Urick ME, Bell DW. Clinical actionability of molecular targets in endometrial cancer. Nat Rev Cancer. 2019;19:510-21.

2. Guo C, Zhao W, Bao X-F. LncRNA NNT-AS1 affects progesterone resistance by regulating MiR-542-3p/survivin axis in endometrial. Cancer Clin Surg Res Commun. 2019;3:23-35.

3. Brooks RA, Fleming GF, Lastra RR, Lee NK, Moroney JW, Son $\mathrm{CH}$, et al. Current recommendations and recent progress in endometrial cancer. CA: Cancer J Clin. 2019;69:258-79.

4. Shinde A, Li R, Amini A, Chen YJ, Cristea M, Dellinger T, et al. Improved survival with adjuvant brachytherapy in stage IA endometrial cancer of unfavorable histology. Gynecologic Oncol. 2018;151:82-90.

5. Hsieh HY, Wang L, Lu CH, Lin JC, Chen CC. Role of adjuvant radiotherapy in FIGO stage IIIC endometrial carcinoma: treatment outcomes and prognostic factors in 52 irradiated patients. J Formos Med Assoc. 2018;117:613-20.

6. Sorolla MA, Parisi E, Sorolla A. Determinants of sensitivity to radiotherapy in endometrial cancer. Cancers. 2020;12:1906.

7. Gu S, Ni T, Wang J, Liu Y, Fan Q, Wang Y, et al. CD47 blockade inhibits tumor progression through promoting phagocytosis of tumor cells by $\mathrm{M} 2$ polarized macrophages in endometrial cancer. J Immunol Res. 2018;2018:6156757.

8. Li LY, Yin KM, Bai YH, Zhang ZG, Di W, Zhang S. CTHRC1 promotes M2-like macrophage recruitment and myometrial invasion in endometrial carcinoma by integrin-Akt signaling pathway. Clin Exp Metastas. 2019;36:351-63.

9. Meng $\mathrm{Y}$, Beckett MA, Liang $\mathrm{H}$, Mauceri HJ, van Rooijen $\mathrm{N}$, Cohen KS, et al. Blockade of tumor necrosis factor alpha signaling in tumor-associated macrophages as a radiosensitizing strategy. Cancer Res. 2010;70:1534-43.

10. Wortzel I, Dror S, Kenific CM, Lyden D. Exosome-mediated metastasis: communication from a distance. Dev Cell. 2019;49:347-60.

11. Feng Z, Zhang $X$, Li L, Wang C, Feng M, Zhao K, et al. Tumor-associated macrophage-derived exosomal microRNA-155-5p stimulates intracranial aneurysm formation and macrophage infiltration. Clin Sci. 2019;133:2265-82.

12. Zheng $P$, Chen $L$, Yuan $X$, Luo Q, Liu Y, Xie G, et al. Exosomal transfer of tumorassociated macrophage-derived miR-21 confers cisplatin resistance in gastric cancer cells. J Exp Clin Cancer Res. 2017;36:53.

13. Liu J, Xue N, Guo Y, Niu K, Gao L, Zhang S, et al. CircRNA_100367 regulated the radiation sensitivity of esophageal squamous cell carcinomas through miR-217/ Wnt3 pathway. Aging. 2019;11:12412-27.

14. Yang J, Zhu D, Liu S, Shao M, Liu Y, Li A, et al. Curcumin enhances radiosensitization of nasopharyngeal carcinoma by regulating circRNA network. Mol Carcinog. 2020;59:202-14. 
15. Li H, Zhi Y, Ma C, Shen Q, Sun F, Cai C. Circ_0062020 knockdown strengthens the radiosensitivity of prostate cancer cells. Cancer Manag Res. 2020;12:11701-12.

16. Xu H, Gong Z, Shen Y, Fang Y, Zhong S. Circular RNA expression in extracellular vesicles isolated from serum of patients with endometrial cancer. Epigenomics. 2018;10:187-97.

17. Cassetta L, Noy R, Swierczak A, Sugano G, Smith H, Wiechmann L, et al. Isolation of mouse and human tumor-associated macrophages. Adv Exp Med Biol. 2016;899:211-29.

18. Zhang $Y, Y u$ G, Chu H, Wang $X$, Xiong L, Cai G, et al. Macrophage-associated PGK1 phosphorylation promotes aerobic glycolysis and tumorigenesis. Mol Cell. 2018;71:201-15.e7.

19. Huang $\mathrm{P}$, Wang $\mathrm{L}, \mathrm{Li} \mathrm{Q}$, Tian $\mathrm{X}, \mathrm{Xu} J, \mathrm{Xu} \mathrm{J}$, et al. Atorvastatin enhances the therapeutic efficacy of mesenchymal stem cells-derived exosomes in acute myocardial infarction via up-regulating long non-coding RNA H19. Cardiovascular Res. 2020;116:353-67.

20. Roman MG, Flores LC, Cunningham GM, Cheng C, Dube S, Allen C, et al. Thioredoxin overexpression in mitochondria showed minimum effects on aging and age-related diseases in male C57BL/6 mice. Aging Pathobiol Ther. 2020;2:20-31.

21. Ji RC. Macrophages are important mediators of either tumor- or inflammationinduced lymphangiogenesis. Cell Mol Life Sci. 2012;69:897-914.

22. Rebelo SP, Pinto C, Martins TR, Harrer N, Estrada MF, Loza-Alvarez P, et al. 3D-3culture: a tool to unveil macrophage plasticity in the tumour microenvironment. Biomaterials. 2018;163:185-97.

23. Zhao R, Ying M, Gu S, Yin W, Li Y, Yuan $H$, et al. Cysteinyl leukotriene receptor 2 is involved in inflammation and neuronal damage by mediating microglia M1/M2 polarization through NF-KB pathway. Neuroscience. 2019;422:99-118.

24. Benson MJ, Manzanero S, Borges K. Complex alterations in microglial M1/M2 markers during the development of epilepsy in two mouse models. Epilepsia. 2015;56:895-905.

25. Zheng $P$, Luo $Q$, Wang W, Li J, Wang T, Wang $P$, et al. Tumor-associated macrophages-derived exosomes promote the migration of gastric cancer cells by transfer of functional Apolipoprotein E. Cell Death Dis. 2018;9:434.

26. Kwon Y, Kim M, Kim Y, Jung HS, Jeoung D. Exosomal MicroRNAs as mediators of cellular interactions between cancer cells and macrophages. Front Immunol. 2020;11:1167.

27. Yang $R$, Liao $Y$, Wang $L, H e P, H u Y$, Yuan $D$, et al. Exosomes derived from $M 2 b$ macrophages attenuate DSS-induced colitis. Front Immunol. 2019;10:2346.

28. Liu KS, Pan F, Mao XD, Liu C, Chen YJ. Biological functions of circular RNAs and their roles in occurrence of reproduction and gynecological diseases. Am J Transl Res. 2019;11:1-15.

29. Wang Y, Liu J, Ma J, Sun T, Zhou Q, Wang W, et al. Exosomal circRNAs: biogenesis, effect and application in human diseases. Mol Cancer. 2019;18:116.

30. Shen Q, He T, Yuan H. Hsa_circ_0002577 promotes endometrial carcinoma progression via regulating miR-197/CTNND1 axis and activating Wnt/B-catenin pathway. Cell Cycle. 2019;18:1229-40.

31. Zong ZH, Liu Y, Chen S, Zhao Y. Circ_PUM1 promotes the development of endometrial cancer by targeting the miR-136/NOTCH3 pathway. J Cell Mol Med. 2020;24:4127-35.

32. Liu Y, Chen S, Zong ZH, Guan X, Zhao Y. CircRNA WHSC1 targets the miR-646/ NPM1 pathway to promote the development of endometrial cancer. J Cell Mol Med. 2020;24:6898-907.

33. Zhou Y, Shen L, Wang YZ, Zhou CC. The potential of ciRS-7 for predicting onset and prognosis of cervical cancer. Neoplasma. 2020;67:312-22.

34. Liu Y, Chang Y, Cai Y. Hsa_circ_0061140 promotes endometrial carcinoma progression via regulating miR-149-5p/STAT3. Gene. 2020;745:144625.

35. Li Q, Li N, Liu H, Du Y, He H, Zhang L, et al. Estrogen-decreased hsa_circ_0001649 promotes stromal cell invasion in endometriosis. Reproduction. 2020;160:511-9.

36. Ye F, Tang QL, Ma F, Cai L, Chen M, Ran XX, et al. Analysis of the circular RNA transcriptome in the grade 3 endometrial cancer. Cancer Manag Res. 2019:11:6215-27.

37. Song W, Guo C, Chen J, Duan S, Hu Y, Zou Y, et al. Silencing PSME3 induces colorectal cancer radiosensitivity by downregulating the expression of cyclin B1 and CKD1. Exp Biol Med. 2019;244:1409-18.

38. Wang L, Xia Y, Chen T, Zeng Y, Li L, Hou Y, et al. Sanyang Xuedai enhances the radiosensitivity of human non-small cell lung cancer cells via increasing iNOS/NO production. Biomed Pharmacother. 2018;102:618-25.

39. Xie B, Wang S, Jiang N, Li JJ. Cyclin B1/CDK1-regulated mitochondrial bioenergetics in cell cycle progression and tumor resistance. Cancer Lett. 2019;443:56-66.

40. Juríková M, Danihel L', Polák Š, Varga I. Ki67, PCNA, and MCM proteins: markers of proliferation in the diagnosis of breast cancer. Acta Histochem. 2016;118:544-52.

41. Salmaninejad A, Valilou SF, Soltani A, Ahmadi S, Abarghan YJ, Rosengren RJ, et al. Tumor-associated macrophages: role in cancer development and therapeutic implications. Cell Oncol. 2019;42:591-608.
42. Lin $Y, X u J$, Lan $\mathrm{H}$. Tumor-associated macrophages in tumor metastasis: biological roles and clinical therapeutic applications. J Hematol Oncol. 2019;12:76.

43. Ozpiskin OM, Zhang L, Li JJ. Immune targets in the tumor microenvironment treated by radiotherapy. Theranostics. 2019;9:1215-31.

44. Darragh LB, Oweida AJ, Karam SD. Overcoming resistance to combination radiation-immunotherapy: a focus on contributing pathways within the tumor microenvironment. Front Immunol. 2018;9:3154.

45. Niu Y, Zhou B, Wan C, Wu R, Sun H, Lu D. Down-regulation of miR-181a promotes microglial M1 polarization through increasing expression of NDRG2. Aging Pathobiol Ther. 2020;2:52-7.

46. Chen Y, Song Y, Du W, Gong L, Chang H, Zou Z. Tumor-associated macrophages: an accomplice in solid tumor progression. J Biomed Sci. 2019;26:78.

47. Valeta-Magara A, Gadi A, Volta V, Walters B, Arju R, Giashuddin S, et al. Inflammatory breast cancer promotes development of M2 tumor-associated macrophages and cancer mesenchymal cells through a complex chemokine network. Cancer Res. 2019;79:3360-71.

48. Yamaguchi T, Fushida S, Yamamoto $Y$, Tsukada T, Kinoshita J, Oyama K, et al. Tumor-associated macrophages of the M2 phenotype contribute to progression in gastric cancer with peritoneal dissemination. Gastric Cancer. 2016;19:1052-65.

49. Tariq M, Zhang JQ, Liang GK, He QJ, Ding L, Yang B. Gefitinib inhibits M2-like polarization of tumor-associated macrophages in Lewis lung cancer by targeting the STAT6 signaling pathway. Acta Pharmacol Sin. 2017:38:1501-11.

50. Shiao SL, Ruffell B, DeNardo DG, Faddegon BA, Park CC, Coussens LM. TH2polarized CD4(+) T cells and macrophages limit efficacy of radiotherapy. Cancer Immunol Res. 2015;3:518-25.

51. Xu J, Escamilla J, Mok S, David J, Priceman S, West B, et al. CSF1R signaling blockade stanches tumor-infiltrating myeloid cells and improves the efficacy of radiotherapy in prostate cancer. Cancer Res. 2013;73:2782-94.

52. Tai YL, Chen KC, Hsieh JT, Shen TL. Exosomes in cancer development and clinical applications. Cancer Sci. 2018;109:2364-74.

53. Pawlik TM, Keyomarsi K. Role of cell cycle in mediating sensitivity to radiotherapy. Int J Radiat Oncol Biol Phys. 2004;59:928-42.

54. Zhang SX, Qiu QH, Chen WB, Liang $\mathrm{CH}$, Huang B. Celecoxib enhances radiosensitivity via induction of $\mathrm{G}_{2}-\mathrm{M}$ phase arrest and apoptosis in nasopharyngeal carcinoma. Cell Physiol Biochem. 2014;33:1484-97.

55. Pradier O, Rave-Fränk M, Lehmann J, Lücke E, Boghun O, Hess CF, et al. Effects of docetaxel in combination with radiation on human head and neck cancer cells (ZMK-1) and cervical squamous cell carcinoma cells (CaSki). Int J Cancer. 2001;91:840-5.

56. Wang J, Chang L, Lai X, Li X, Wang Z, Huang Z, et al. Tetrandrine enhances radiosensitivity through the $C D C 25 C / C D K 1 /$ cyclin $B 1$ pathway in nasopharyngeal carcinoma cells. Cell Cycle. 2018;17:671-80.

57. Tan C, Qian X, Ge Y, Yang B, Wang F, Guan Z, et al. Oroxylin a could be a promising radiosensitizer for esophageal squamous cell carcinoma by inducing G2/M arrest and activating apoptosis. Pathol Oncol Res. 2017;23:323-8.

58. Dukaew N, Konishi T, Chairatvit K, Autsavapromporn N, Soonthornchareonnon N, Wongnoppavich A. Enhancement of radiosensitivity by eurycomalactone in human NSCLC cells through $\mathrm{G}_{2} / \mathrm{M}$ cell cycle arrest and delayed DNA doublestrand break repair. Oncol Res. 2020;28:161-75.

59. Lv S, Xu X, Wu Z. Identification of key candidate genes and pathways in endometrial cancer: evidence from bioinformatics analysis. Oncol Lett. 2019;18:6679-89.

\section{AUTHOR CONTRIBUTIONS}

XBG: conceptualization, methodology, writing-original draft; YGS and MLD: investigation; LJ and JY: data curation; ZYL: writing—reviewing and editing.

\section{FUNDING}

This study was supported by the Joint Construction Project of Medical Science and Technology Research Program of Henan Province (No. LHGJ20190019).

\section{COMPETING INTERESTS}

The authors declare no competing interests.

\section{ETHICS APPROVAL}

All patients signed informed consent forms. This investigation involving human specimens was approved by the Ethics Committee of The First Affiliated Hospital of Zhengzhou University. All animal experiments were approved by the Committee on the Ethics of The First Affiliated Hospital of Zhengzhou University. 


\section{ADDITIONAL INFORMATION}

Supplementary information The online version contains supplementary material available at https://doi.org/10.1038/s41419-021-04087-8.

Correspondence and requests for materials should be addressed to X.G.

Reprints and permission information is available at http://www.nature.com/ reprints

Publisher's note Springer Nature remains neutral with regard to jurisdictional claims in published maps and institutional affiliations.
Open Access This article is licensed under a Creative Commons cc) Attribution 4.0 International License, which permits use, sharing,
adaptation, distribution and reproduction in any medium or format, as long as you give appropriate credit to the original author(s) and the source, provide a link to the Creative Commons license, and indicate if changes were made. The images or other third party material in this article are included in the article's Creative Commons license, unless indicated otherwise in a credit line to the material. If material is not included in the article's Creative Commons license and your intended use is not permitted by statutory regulation or exceeds the permitted use, you will need to obtain permission directly from the copyright holder. To view a copy of this license, visit http://creativecommons. org/licenses/by/4.0/.

(c) The Author(s) 2021 